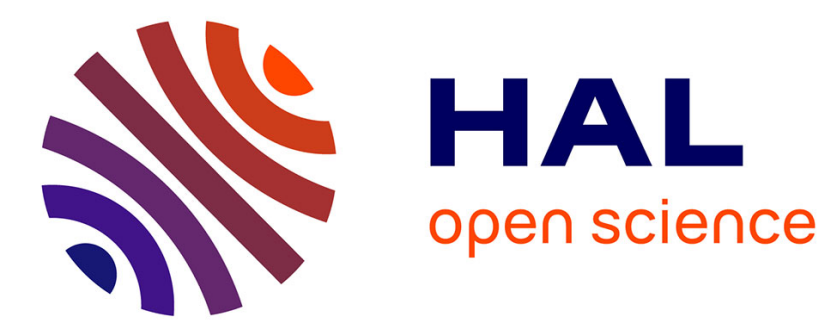

\title{
Benchmarking of monolithic MDO formulations and derivative computation techniques using OpenMDAO
}

Scott Delbecq, Marc Budinger, Aurélien Reysset

\section{To cite this version:}

Scott Delbecq, Marc Budinger, Aurélien Reysset. Benchmarking of monolithic MDO formulations and derivative computation techniques using OpenMDAO. Structural and Multidisciplinary Optimization, 2020, 62 (2), pp.645-666. 10.1007/s00158-020-02521-7 . hal-02997582

\section{HAL Id: hal-02997582 \\ https://hal.science/hal-02997582}

Submitted on 10 Nov 2020

HAL is a multi-disciplinary open access archive for the deposit and dissemination of scientific research documents, whether they are published or not. The documents may come from teaching and research institutions in France or abroad, or from public or private research centers.
L'archive ouverte pluridisciplinaire HAL, est destinée au dépôt et à la diffusion de documents scientifiques de niveau recherche, publiés ou non, émanant des établissements d'enseignement et de recherche français ou étrangers, des laboratoires publics ou privés. 


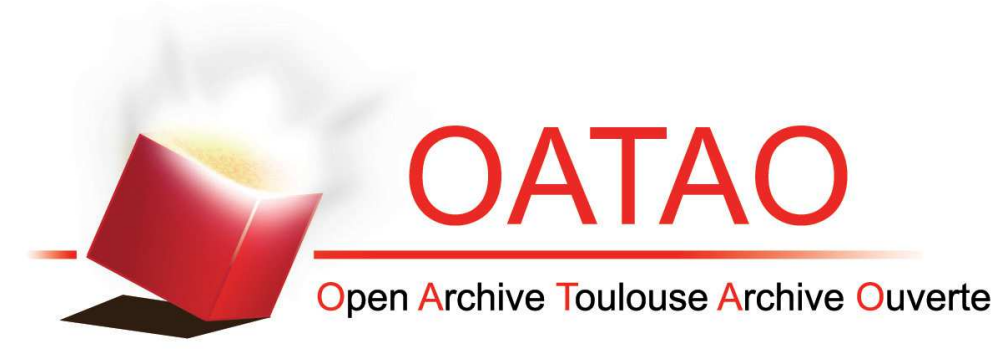

\section{Open Archive Toulouse Archive Ouverte (OATAO)}

OATAO is an open access repository that collects the work of some Toulouse researchers and makes it freely available over the web where possible.

This is an author's version published in: https://oatao.univ-toulouse.fr/26689

Official URL: https://doi.org/10.1007/s00158-020-02521-7

\section{To cite this version :}

Delbecq, Scott and Budinger, Marc and Reysset, Aurélien Benchmarking of monolithic MDO formulations and derivative computation techniques using OpenMDAO. (2020) Structural and Multidisciplinary Optimization, 62 (2). 645-666. ISSN 1615-147X

Any correspondence concerning this service should be sent to the repository administrator: tech-oatao@listes-diff.inp-toulouse.fr 


\title{
Benchmarking of monolithic MDO formulations and derivative computation techniques using OpenMDAO
}

\author{
Scott Delbecq ${ }^{1}$ (D) Marc Budinger ${ }^{2} \cdot$ Aurélien Reysset $^{2}$
}

\begin{abstract}
The design optimization of coupled systems requires the implementation of multidisciplinary design optimization techniques in order to obtain consistent and optimal solutions. The associated research topics include the development of optimization algorithms, computational frameworks, and multidisciplinary design optimization formulations. This paper presents a benchmarking of the combination of monolithic formulations and derivative computation techniques. The monolithic formulations include typical literature formulations as well as new normalized variable hybrid formulation. A novel test problem is proposed which consists in the sizing of a space launcher thrust vector control electro-mechanical actuator. Solving the single multidisciplinary coupling present in this problem is complex due to the possibility to face one, two, or no solutions depending on the external load and reducer gear ratio configuration. A larger scale version of this test problem is also proposed and tested by adding a high degree of freedom point-to-point trajectory. The tests are carried out in order to obtain typical performance criteria but also some proposed additional robustness criteria such as variation of the initial conditions or the external load scale. These additional criteria are particularly relevant in an industrial engineering design context where knowledge capitalization and reuse are sought. The most significant findings are the interesting performances of the new formulation in terms of computational cost and the robustness. Furthermore, the effect of the choice of derivative computation strategy on different performance criteria is assessed for the original and larger scale problem, and thus underlines the benefits of full analytic gradient-based optimization.
\end{abstract}

Keywords Multidisciplinary design optimization - Coupled systems · Sizing · Robustness · Electro-mechanical actuator · Thrust vector control

\section{Introduction}

Engineering design activities can gain productivity and product performance through the practice of design

\author{
Responsible Editor: Raphael Haftka \\ Scott Delbecq \\ scott.delbecq@isae-supaero.fr \\ Marc Budinger \\ marc.budinger@insa-toulouse.fr \\ Aurélien Reysset \\ aurelien.reysset@insa-toulouse.fr \\ 1 Department of Aerospace Vehicles Design and Control, \\ ISAE-SUPAERO, Université de Toulouse, Toulouse, France \\ 2 Department of Mechanical Engineering, Université \\ de Toulouse, UPS, INSA, ISAE-SUPAERO, MINES-ALBI, \\ CNRS, ICA, Toulouse, France
}

optimization based on the theory and the implementation of numerical optimization. For the regular engineer individual, the difficulty is to choose and master the adequate methods and tools that facilitate the problem implementation. The problem implementation includes two aspects. The first is the formulation of the design problem:"to obtain an optimal product what should one set as a the design variables, the constraints, the objective in his sizing code?" Secondly, is the aspects regarding the methods and tools one should use: "which algorithm? which method to assess constraints? in what environment? is differentiation possible in the sizing code?" An additional difficulty for engineers is to deal with multidisciplinary couplings that are commonly present in engineering design problems. Also, a worry for engineers will be the capability of the code to be reused for different system requirements with the least redevelopment or tuning effort. Reuse is a key to capitalization and thus time gain but it is strongly dependent on what will be referred to as in this paper the robustness of the code. Hence, three main 
interest criteria can be underlined for an engineering when attempting to implement a numerical optimization problem:

- Easiness of the implementation

- Performance (computational cost, convergence...)

- Robustness (initial conditions, scale change...)

The scientific literature seeks to help assess these criteria for different numerical optimization methods in order to enable individuals pick the adequate method regarding their needs and what compels them. Most research focus on the assessment of performance aspects with criteria such as computation time (Chell et al. 2019), number of evaluations (Tedford and Martins 2010; Gray et al. 2013), convergence characteristics (Tedford and Martins 2010; Gray et al. 2013), or reliability (Beiranvand et al. 2017).

In the field of multidisciplinary design optimization (MDO), many benchmarks focus on the comparison of the MDO formulations, also referred to as architectures (Martins and Lambe 2013). The comparison is often made for a set of distributed formulations (collaborative optimization, CO; concurrent subspace optimization, CSSO; Bi-Level Integrated System Synthesis, BLISS) and monolithic formulations (Multidisciplinary Feasible, MDF; Individual Design Feasible, IDF; Simultaneous Analysis and Design, SAND) (Kodiyalam and Sobieszczanski-Sobieski 2001; Martins and Lambe 2013; Gray et al. 2013). It is also interesting to have a look at the derivative computation techniques for the application of gradient-based optimization to MDO or Multidisciplinary Design Analysis (MDA). Gray et al. (2013) compared the effect of computing derivatives analytically or numerically in the field of MDO. Conversely, Chauhan et al. (2017) evaluated jointly the effectiveness of derivative computation techniques and system analyzer for MDA purposes.

Test cases are important resources for benchmarking in numerical optimization (Beiranvand et al. 2017). For MDO formulations benchmarking, one can often find analytic test cases such as the Sellar problem (Sellar et al. 1996) and scalable problem (Martins and Hwang 2013; Gray et al. 2013) as well as some more practical engineering design problems such as the speed reducer (Tedford and Martins 2010) or electrical packaging design (Kodiyalam and Sobieszczanski-Sobieski 2001). Larger engineering design problems are tackled as well such as aircraft conceptual design (Perez et al. 2004; Chell et al. 2019) or reusable launcher design (Kodiyalam and Sobieszczanski-Sobieski 2001).

In most cases, benchmarkings in the field of Multidisciplinary Design Analysis and Optimization (MDAO) evaluate separately the MDO and MDA purposes. In addition, formulations and the derivative computation strategies are often compared independently. Also, one can often find the same set of formulations or example test problems. Furthermore, few literatures investigate choice criteria such as robustness or reliability performance as well as aspect regarding the easiness of the implementation.

This paper aims at addressing these points by proposing a large set of test configurations that includes the derivative computation strategy, the MDO formulation, and the purpose (MDO or MDA). Here, a focus is made on monolithic formulations where a formulation present in the literature is included, the HYBRID, and a new formulation that is proposed, the normalized variable hybrid $(\mathrm{NVH})$, in additional to two typical formulations (MDF and IDF). The performance criteria include some typical ones such as relative error, number of evaluations, and convergence characteristics. This paper also includes novel criteria regarding robustness by investigating the effect of initial conditions variations, scale variations, and design space exploration purposes. Finally, one can also find a novel test problem from the field of engineering design, the optimal sizing of a high dynamic electro-mechanical actuator.

The most significant findings are the interesting performances of the new NVH formulation in terms of computational cost and the robustness to initial conditions and scale changes of the external load and therefore enables more reusable sizing codes. Furthermore, the effect of the choice of derivative computation strategy on different performance criteria is assessed and underlines the benefits of full analytic gradient-based optimization.

To present the proposed approach, the paper is organized as follows. Section 2 describes the proposed test problem and the associated challenges. Also, the implementation of MDO formulations chosen is given. Finally, a larger scale version of the EMA design problem is depicted. Section 3 presents the tests related to the EMA design problem. First, typical test performance is driven and the obtained results are outlined. Then, additional tests regarding robustness are ran and the associated results analyzed. In Section 4, similar tests are performed on the larger scale EMA design problem and differences with the original problem underlined. Section 5 extends some of the benchmarking to the Sellar problem and shows some recent applications of the NVH formulation. Finally, Section 6 offers concluding remarks.

\section{The electro-mechanical actuator design problem}

\subsection{Problem description}

The case study presented here is a simple design optimization problem that illustrates the multidisciplinary design of 
a mechatronic device. The low number of analysis functions required to represent the problem makes it a convenient test problem for MDO formulation benchmarking. The studied system is an electro-mechanical actuator designed for dynamic applications such as an Individual Blade Control (IBC) (Kessler 2011) or Thrust Vector Control (TVC) (Carnevale and Resta 2007). Despite the simplicity in terms of number of analysis functions, the design of an electromechanical actuator for TVC aerospace application is still a challenging topic. Figure 1 shows that for launchers, electro-hydraulic actuators were preferred because of the high dynamic and high loading conditions.

Nevertheless, new permanent magnet motors have made the use of electro-mechanical actuators (EMAs) a success on small launchers (Stefano Bianchi 2005). However, such applications are on the technological limit frontier of such systems. For some requirements, there might be no existing solution because of these technological constraints. Hence, the numerical resolution of the sizing problem for TVC electro-mechanical actuators is challenging.

The EMA is composed of a ball screw, a spur gear set, and a brushless motor as shown in Fig. 2. The maximum external load acting on the actuator and the maximum acceleration and speed of the actuator have been extracted from the mission profile. Since the application is high dynamic, the effect of motor inertial acceleration has to be considered when choosing the motor torque performances. The sizing problem is expressed using only algebraic analysis functions. For more complex design problems where numerical simulations are necessary (Delbecq 2018), it is possible to reduce them to algebraic

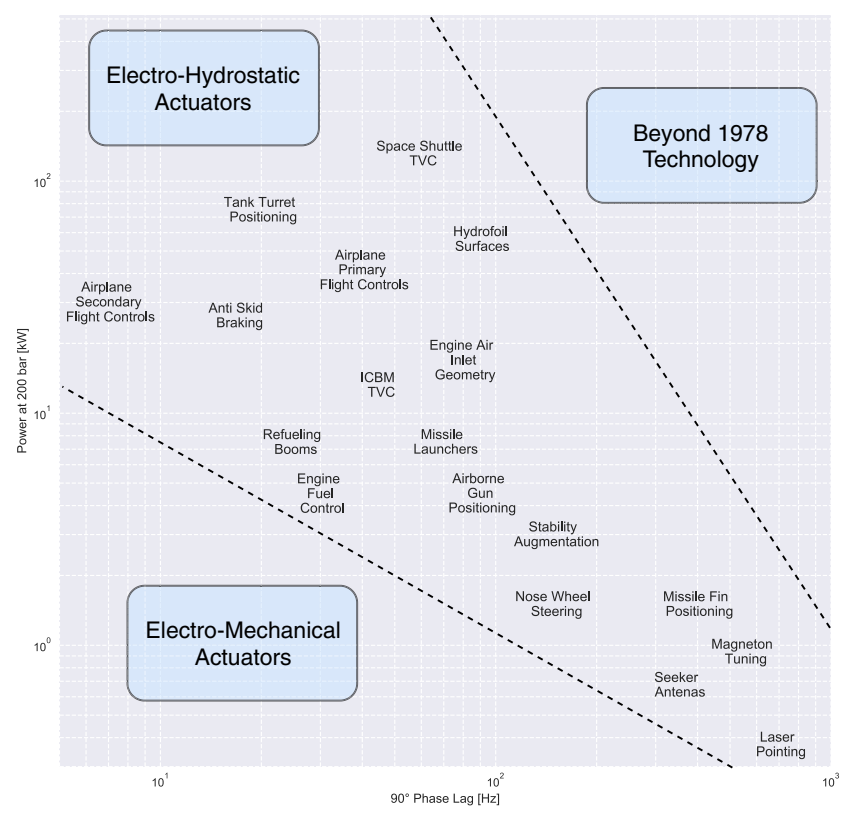

Fig. 1 Spectrum of Servoactuation Aerospace Applications in 1978 adapted from Maskrey and Thayer (1978)

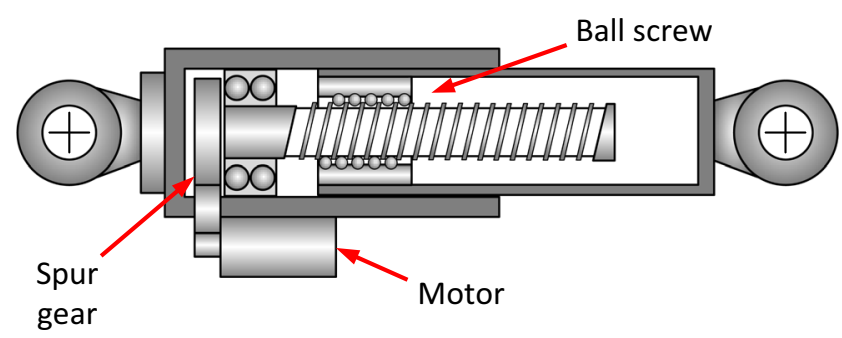

Fig. 2 High dynamic EMA architecture

functions using surrogate modelling techniques. These techniques have been successfully used to replace lumped parameter (0D-1D) models, e.g., 2D multibody simulation for estimating the actuator load with respect to the attachment locations (Budinger et al. 2014), or finite element method (3D) models, e. g., 3D electromagnetic FEM model for estimating the motor torque with respect to its geometry (Sanchez et al. 2017b).

The EMA sizing problem is relatively simple to implement but includes numerical difficulties which make its resolution challenging. The problem is represented by the following equations and inequalities:

$T_{\text {em }}=J_{\text {mot }} \cdot A_{\text {max }} \frac{N_{\text {red }}}{p}+F_{\text {ema }} \frac{p}{N_{\text {red }}}$

$J_{m o t}=J_{m o t_{r e f}} \cdot\left(\frac{T_{e m}}{T_{e m_{r e f}}}\right)^{\frac{5}{3.5}}$

$\Omega_{m o t}=\Omega_{m o t_{r e f}} \cdot\left(\frac{T_{e m}}{T_{e m_{r e f}}}\right)^{-\frac{1}{3.5}}$

$\Omega_{\text {mot }} \geq V_{\text {max }} \cdot \frac{N_{\text {red }}}{p}$

$M_{m o t}=M_{m o t_{r e f}} \cdot\left(\frac{T_{e m}}{T_{e m_{r e f}}}\right)^{\frac{3}{3.5}}$

where $T_{e m}$ is the motor electromagnetic torque, $J_{m o t}$ is the motor inertia, $A_{\max }$ is the maximum actuator acceleration, $N_{\text {red }}$ is the reducer gear ratio, $p$ is the screw pitch, $V_{\max }$ and $F_{\text {ema }}$ are the maximum velocity and external load of the actuator, $\Omega_{m o t}$ the maximum mechanical speed of the motor, and $M_{m o t}$ its mass. The motor sizing scaling laws are based on a reference motor where $T_{\text {mot }_{\text {ref }}}, J_{\text {mot }_{\text {ref }}}$, $\Omega_{\text {mot }_{\text {ref }} \text {, and }} M_{\text {mot }_{\text {ref }}}$ are respectively its electromagnetic torque, inertia, maximal mechanical rotational speed, and mass (Budinger et al. 2014). Motor torque (1) and motor inertia (2) are two coupled disciplines of the sizing problem whereas motor speed (3), motor speed constraint (4), and motor mass objective (5) are ordinary analysis functions. The motor torque (1) and motor inertia (2) are coupled through motor electromagnetic torque $T_{e m}$ and motor inertia 
$J_{m o t}$. The ball screw efficiency is supposed equal to 1 and independent of the pitch and the axial load.

The high-level design problem consists of minimizing the motor mass $M_{m o t}$, with respect to reducer gear ratio $N_{\text {red }}$, subject to motor speed constraint (4).

The optimization problem formulation is the following:

$\begin{array}{ll}\text { minimize } & M_{\text {mot }} \\ \text { with respect to } & N_{\text {red }} \\ \text { subject to } & V_{\text {max }} \cdot \frac{N_{\text {red }}}{p}-\Omega_{\text {mot }} \leq 0\end{array}$

The Extended Design Structure Matrix (XDSM) proposed by Lambe and Martins (2012) is an efficient formalism for representing a design optimization problem and the associated formulation. In this paper, the XDSM diagrams are simplified by removing the numbering of components and the thin black line to streamline the diagrams. Nevertheless, for the considered problems, the execution order and the process flow are deduced from the order of components in XDSM structure as in the N2 chart. The optimization problem, and the multidisciplinary coupling, is represented in Fig. 3 using this formalism.

The analysis or optimization of this problem requests to solve this multidisciplinary coupling. The mathematical definition of the problem makes it possible to have one or two or no solution when attempting to solve the consistency of (1). The number of solutions depends on the configuration in terms of external load $F_{\text {ema }}$ and the reducer gear ratio $N_{\text {red }}$. Figure 4 depicts this particularity by plotting the disciplines represented respectively by (1) and (2).

One can see for instance that $F_{\text {ema }}=200[k N]$ and $N_{\text {red }}=2$ leads to one single existing solution whereas changing the gear ratio to 10 will introduce an additional solution. Conversely, the high load $\left(F_{\text {ema }}=300[\mathrm{kN}]\right)$ and high gear ratio $\left(N_{\text {red }}=10\right)$ configuration has no existing solution. Figure 5 shows the effect of both of these variables on the considered objective $M_{m o t}$. The value of $M_{m o t}$ for configurations where no solution for system consistency exist has been set to 0 . These configurations correspond to high values of external load and reducer gear ratio as shown in Fig. 5.

The multidisciplinary coupling can be solved using different MDO strategies. Each of the strategies is described and represented using the XDSM notation where the mathematical notation is given in Table 1 .

The benchmark of the different multidisciplinary design optimization formulations is achieved using OpenMDAO (Gray et al. 2019). This framework can be used as a standard platform for benchmarking MDO formulations developed by NASA (Gray et al. 2013). Analysis functions are implemented using an explicit form by using the ExplicitComponent class (Gray et al. 2019).

\subsection{The multidisciplinary feasible formulation}

The representation of the MDF strategy for the EMA design problem is given in Fig. 6. It consists of a single design variable, the reducer gear ratio, and a single constraint, the maximum motor speed. The consistency of the coupling between motor torque (1) and motor inertia (2) disciplines is achieved by using a system analyzer. The system analyzer determines the values of coupling variables that match their respective analysis result. Since system analysis is performed for every optimization iteration, couplings variables remain consistent and independent of the optimizer's behavior. Here the first computed discipline is the motor torque that requires an estimation of the motor inertia computed by the subsequent discipline. In such architecture, the decomposition of circular dependencies can be achieved by using Gauss-Seidel or Jacobi iterative methods (Martins and Lambe 2013). Gauss-Seidel uses
Fig. 3 XDSM diagram for the EMA design problem

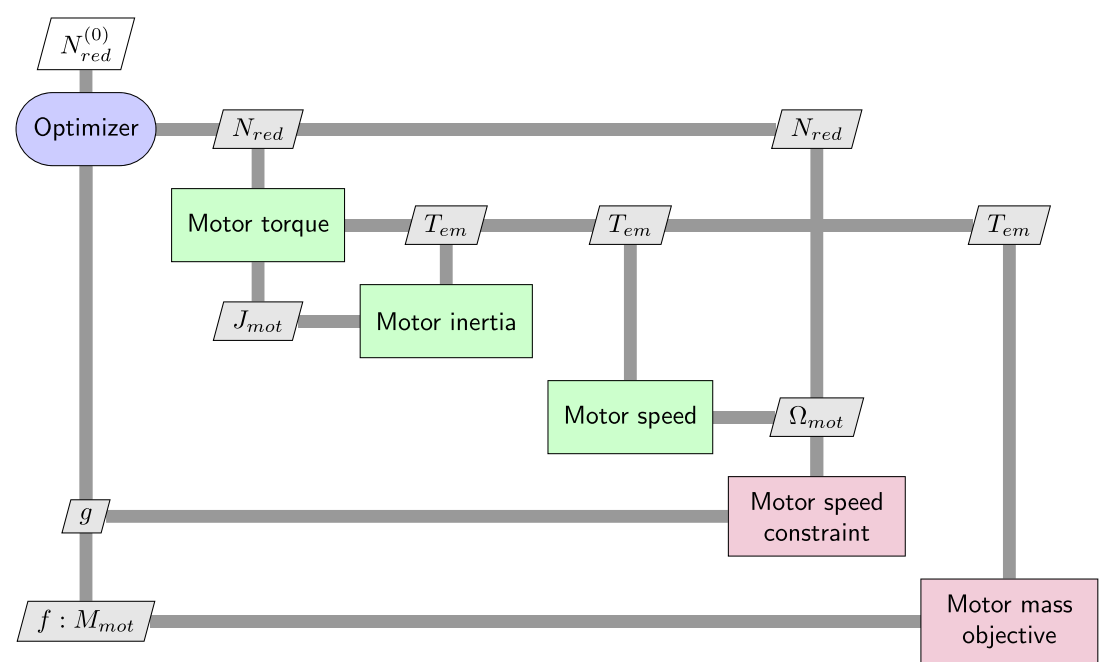




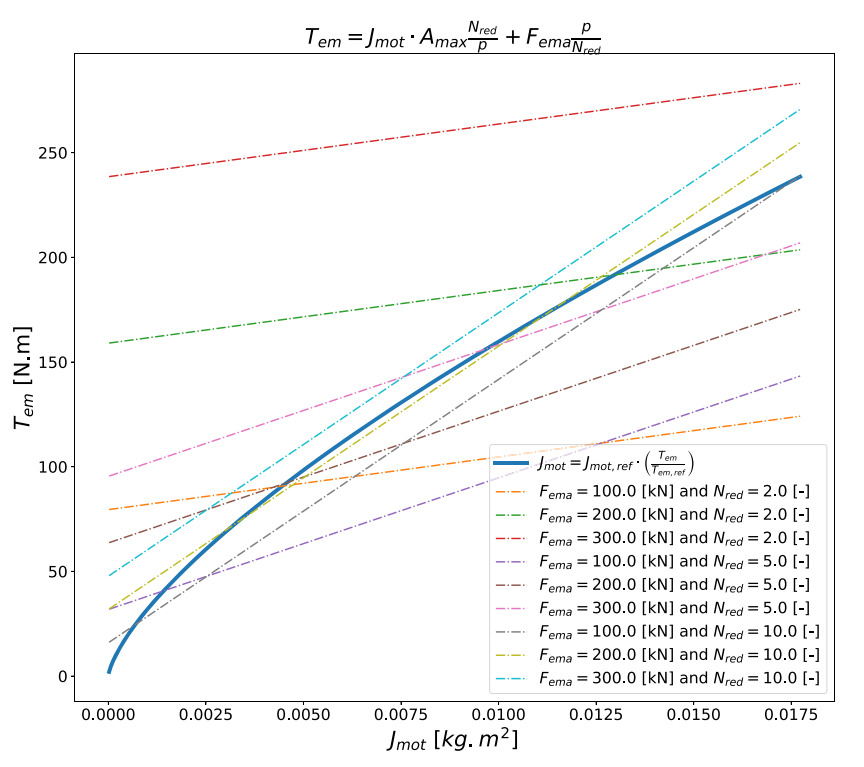

Fig. 4 Zero, one, or two solutions for coupling variables consistency with respect to $F_{\text {ema }}$ and $N_{\text {red }}$

a decomposition into lower and strictly upper triangular elements, whereas the Jacobi method decomposes the linear system into a diagonal element and a corresponding remainder.

The coupling can also be solved by gradient-based solvers like Newton-Raphson. In that case, a linear solver is required to compute coupled derivatives. GaussSeidel or Jacobi methods can be used to obtain coupled derivatives. Another option is to use a non-iterative direct linear solver. It uses the system Jacobian to compute the coupled derivatives using lower-upper (LU) decomposition, which is possible for any non-singular square matrix (Bunch and Hopcroft 1974). The coupled derivatives are also required to compute total system derivatives when

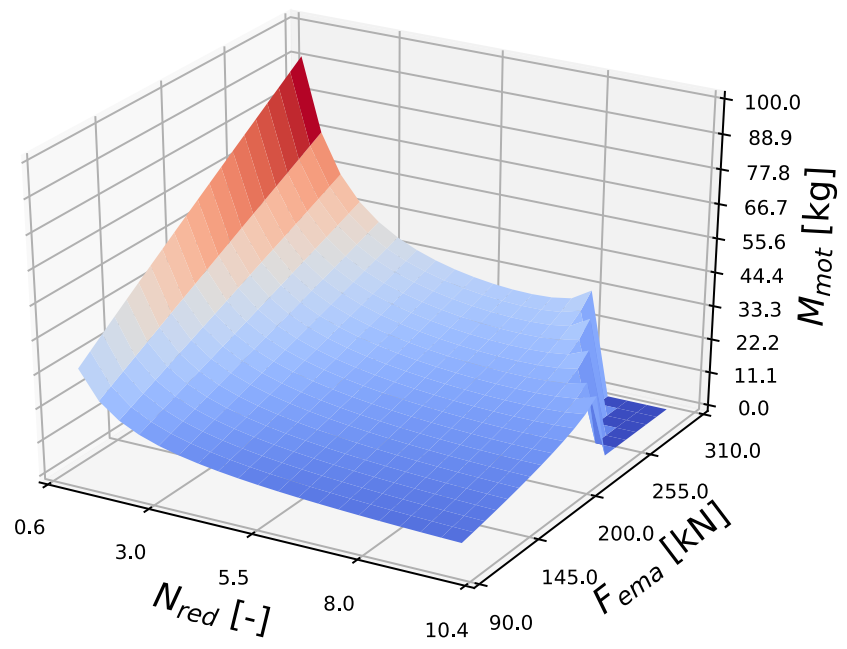

Fig. 5 Illustration of inconsistent solutions $\left(M_{m o t}=0\right)$ in the design space due to the technological limit of EMAs for TVC applications
Table 1 Mathematical notation for MDO problem data (adapted from Lambe and Martins (2012))

\begin{tabular}{ll}
\hline Symbol & Definition \\
\hline$x$ & Vector of design variables \\
$x^{(0)}$ & Vector of design variables initial values \\
$y^{t}$ & Vector of coupling variable targets \\
$y^{t,(0)}$ & Vector of coupling variable targets initial values \\
$y$ & Vector of coupling variable responses \\
$f$ & Objective \\
$g$ & Vector of design constraints \\
$g_{c}$ & Vector of consistency constraints \\
\hline
\end{tabular}

using gradient-based optimizers. All these solvers are implemented within the OpenMDAO framework. Here, a direct linear solver and a Newton non-linear solver perform the multidisciplinary analysis. The choice of adequate solvers is tricky and significantly impacts the effectiveness of the MDA. Choosing the most adapted numerical solver is highly dependent of the expertise and experience of the engineer in MDO problem solving.

The MDF formulation enables us to use no additional design variables or consistency constraints and delivers a consistent design even if the optimizer fails to find a feasible solution and reaches the maximum iteration limit. In addition, MDF eases the incorporation of legacy analysis tools which are often very effective to converge within a particular type of physical discipline. The advantages of system analysis have to be kept in perspective since such approaches rely on the system analyzer effectiveness. Indeed, the non-convergence of the analyzer results in nonconsistent designs despite that in some cases, convergence is achieved. Furthermore, strongly coupled disciplines lead to important number of analyzer iterations and hence a costly system analyses.

In practice, the system analyzer for some samples tries to converge the couplings by using negative electromagnetic torque which make (2) singular. This was observed for different analyzers (Newton and Gauss-Seidel) but also for the HYBRID formulation. Therefore, (2) has to be reformulated into (7) by introducing the absolute function to make sure that the electromagnetic torque remains positive.

$J_{m o t}=J_{\text {mot }_{r e f}} \cdot\left(\frac{\left|T_{e m}\right|}{T_{\text {em } r e f}}\right)^{\frac{5}{3.5}}$

\subsection{The individual disciplinary feasible formulation}

Individual disciplinary feasible (IDF) uses also a single optimizer but an analysis for each individual discipline is employed. As shown in Fig. 7, in this formulation, it is the optimizer that coordinates the interactions between 
Fig. 6 XDSM diagram for MDF formulation of the EMA design problem

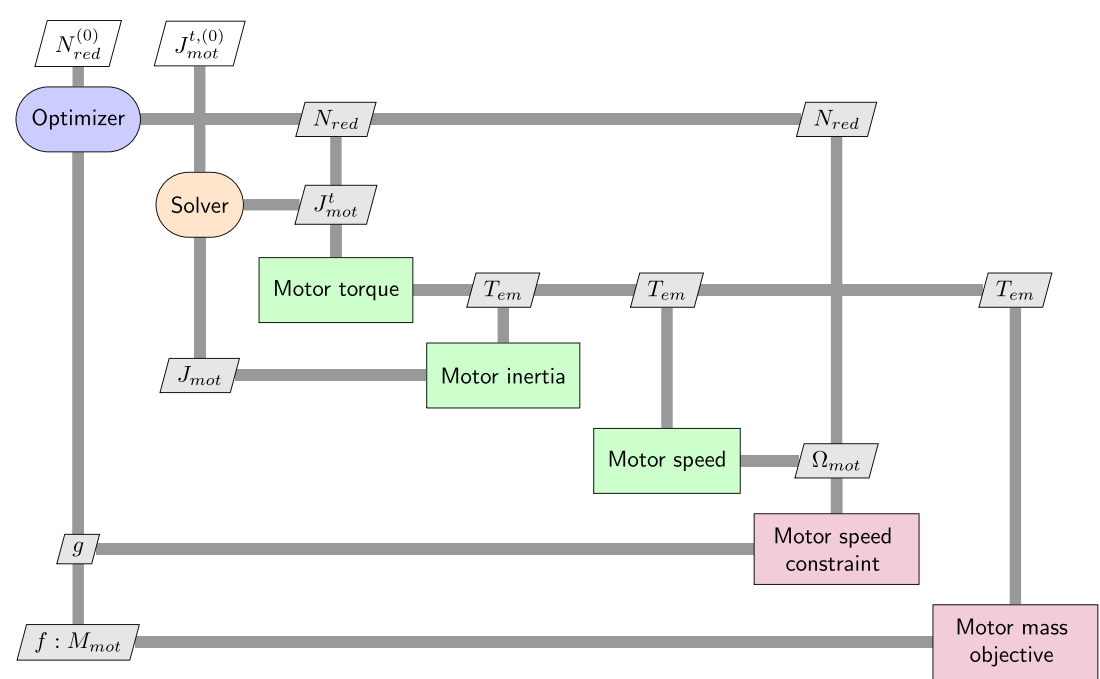

the disciplines analyses. The optimizer chooses values for both design and coupling variables. In order to ensure consistency between coupling variables, additional design variables $T_{e m}, J_{m o t}$ and auxiliary equality constraints are added. Here, the optimizer chooses a value for the motor inertia of (1) and verifies that it is equal to the motor inertia obtained by (2). Similarly, it chooses a value for the motor torque of (2) and then verifies that it is equal to the motor torque obtained by (1). The IDF approach enables us to put aside the intricate concerns of system analyzer effectiveness, but has notable shortcomings. Equality constraints can introduce numerical solution difficulties (Thareja and Haftka 1986) and provide feasibility only at solution, rather than at each iteration (Alexandrov and Lewis 2000). Furthermore, setting bounds on the coupling variables is in some cases not straightforward and can introduce a large dimension of the coupling variables. Large dimension of design variables has a significant effect on the efficiency of the optimization. If the optimization process is interrupted, the design corresponding to the last iteration may be not consistent or feasible whereas MDF guarantees the consistency between coupling variables if the system analyzer is effective.

\subsection{The HYBRID formulation}

Balling and Sobieski proposed a hybrid formulation to handle coupled variables consistency (Balling and Sobieszczanski Sobieski 1996). This architecture is similar to IDF except that, as illustrated in Fig. 8, the feedforward consistency relationship is achieved by computing analysis functions in a sequence, and the feedback consistency relationship remains achieved by an auxiliary equality constraint and an additional design variable $J_{m o t}^{t}$. This
Fig. 7 XDSM diagram for IDF formulation of the EMA design problem

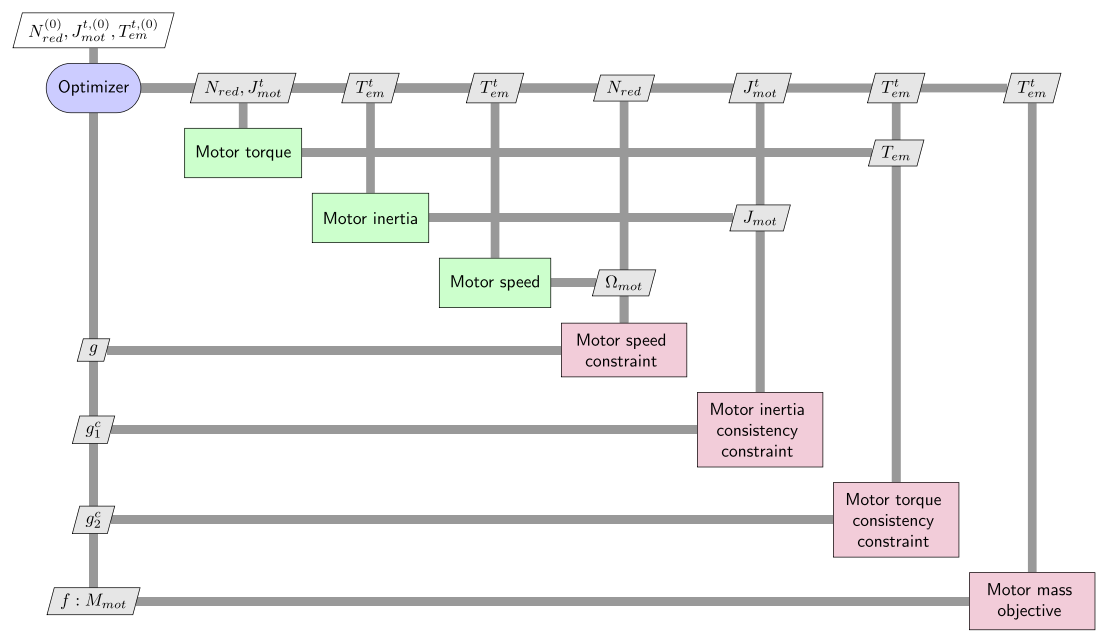


Fig. 8 XDSM diagram for HYBRID formulation of the EMA design problem

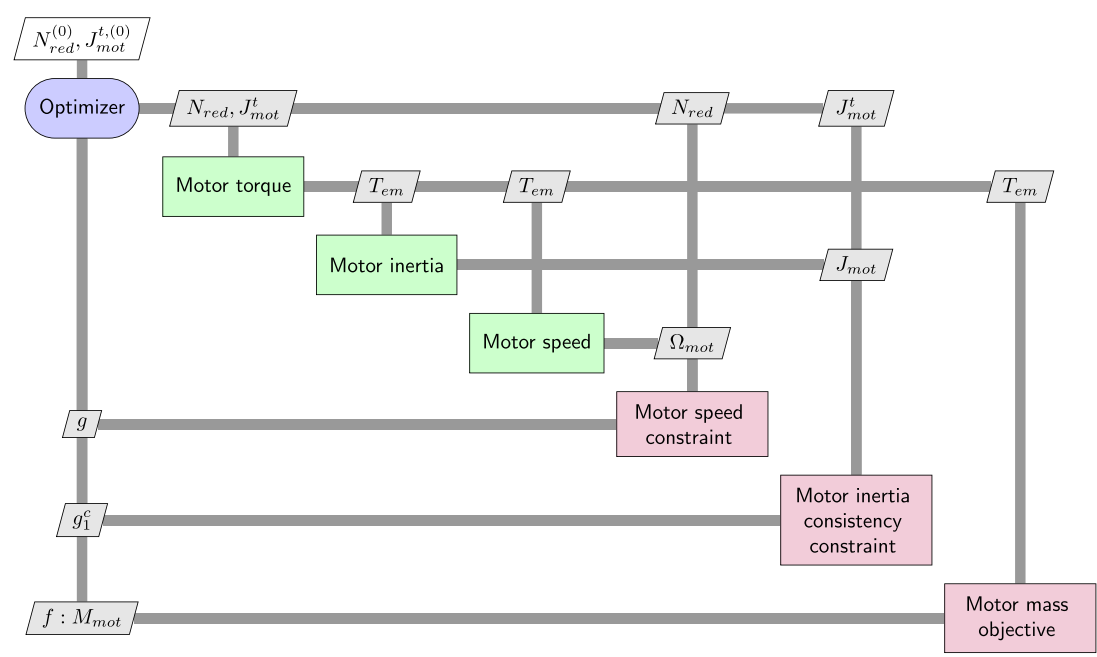

approach has the same advantages and drawbacks as the IDF approach but it enables to remove for the case study one equality constraint and one design variable.

\subsection{The normalized variable hybrid formulation}

The success of attempts to use numerical optimization for design depends strongly on how well the design problem has been formulated. Reysset et al. (2015) proposed to reformulate a multidisciplinary design problem with a view in order to get the best from numerical optimization. This formulation was underlined through a case study but was never benchmarked and compared with other formulations. The NVH formulation, represented in Fig. 9, similarly proposes an evolution of the HYBRID formulation to consider feedforward consistency relationship by computing analysis functions in a set sequence but with a modification of the governing equations. In addition, the feedback consistency relationship is achieved by introducing a normalized design variable and a single inequality constraint. Removing the equality constraint reduces the risk of numerical solution difficulties. Furthermore, the reduction of the new design variable dimension ([1.0-10.0] for most design problems or [0.1-10.0] in particular cases) improves the numerical solution accuracy and the ability to achieve convergence. The difficulty is that this approach requires a reformulation effort which makes it less generic and more invasive than traditional MDO formulations. You must determine how to modify the equations and the form of the new constraint in order to decouple the feedback. Here, discipline (1) is reformulated as in (8), where the normalized design variable is introduced in order to (in this case) oversize the chosen electromagnetic torque. Furthermore, the additional consistency inequality (9) is added to the design problem to verify (in this case) that the chosen electromagnetic torque is sufficient to face the inertial acceleration and the equivalent EMA external load.

$$
T_{e m}=k_{o s} \cdot F_{\text {ema }} \cdot \frac{p}{N_{\text {red }}}
$$

Fig. 9 XDSM diagram for $\mathrm{NVH}$ formulation of the EMA design problem

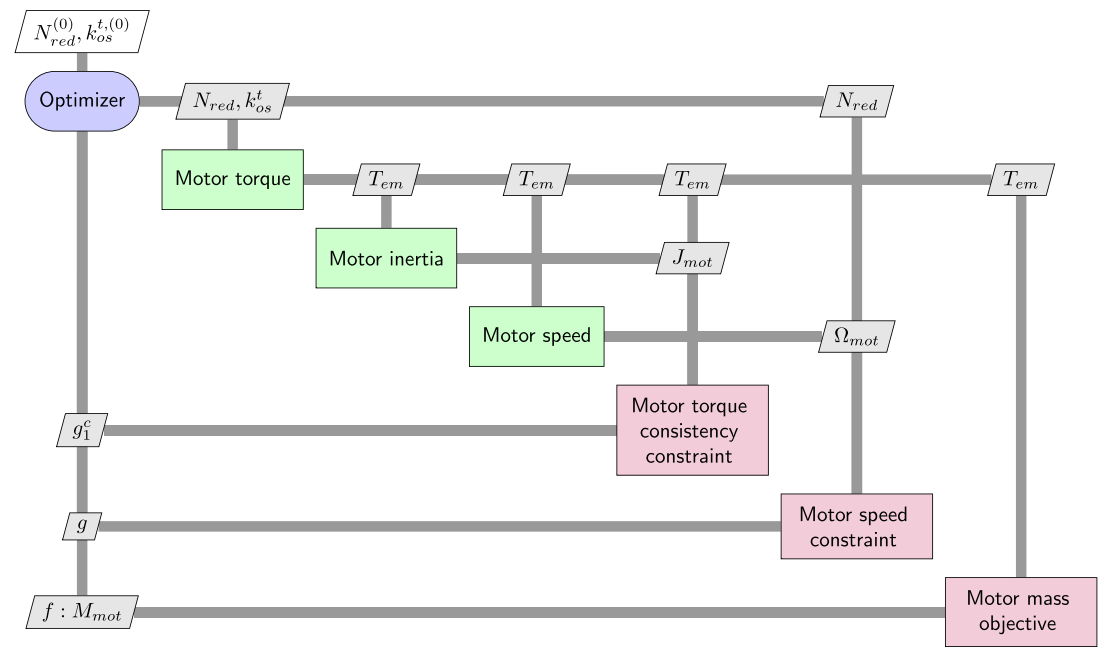


$T_{\text {em }} \geq J_{\text {mot }} \cdot A_{\text {max }} \frac{N_{\text {red }}}{p}+F_{\text {ema }} \frac{p}{N_{\text {red }}}$

The optimization problem becomes

$$
\begin{array}{ll}
\text { min. } & M_{\text {mot }} \\
\text { w.r.t } & N_{\text {red }}, k_{\text {os }} \\
\text { s.t. } & V_{\text {max }} \cdot \frac{N_{\text {red }}}{p}-\Omega_{\text {mot }} \leq 0 \\
& J_{\text {mot }} \cdot A_{\text {max }} \frac{N_{\text {red }}}{p}+F_{\text {ema }} \frac{p}{N_{\text {red }}}-T_{\text {em }} \leq 0 .
\end{array}
$$

A few guidelines to implement the NVH formulation for other problems can be given. The NVH uses a normalized variable $k_{o s}$ which is used to scale the feedforward coupling variable with respect to an initial guess. If one is sure that the final guess is greater than the initial guess, then the lower bound of $k_{o s}$ is equal or greater than 1. Conversely, if one is sure that the final guess is smaller than the initial guess, then the upper bound of $k_{o s}$ is equal or smaller than 1. If one is not sure, the lower bound shall be smaller than 1 and the upper bound greater than 1 . Generally, for most problems, it is straightforward to find an initial guess that limit to [0.1-10.0] as described previously. The most difficult task that enables the NVH to use an inequality instead of an equality as a constraint is that one has to be sure about the effect of $k_{o s}$ on the function objective as the optimizer will seek naturally to make this constraint active. In our case, it is obvious that increasing the motor torque will increase the motor mass. For more complex cases, one may use the monotonicity analysis approach proposed by Papalambros and Wilde (2000).

Table 2 summarizes the different formulation characteristics.

The NVH formulation does not use any equality constraint and only one inequality constraint to solve the coupling. It uses one consistency design variable that has a smaller dimension (in terms of bounds width) when compared with IDF or HYBRID approach. The following section presents some benchmarking results that assess typical performances criteria of each formulation.

Table 2 Formulations characteristics for the larger scale EMA design problem

\begin{tabular}{lllll}
\hline Form. & $\begin{array}{l}\text { Solver } \\
\text { type }\end{array}$ & $\begin{array}{l}\text { Number } \\
\text { of design } \\
\text { variables }\end{array}$ & $\begin{array}{l}\text { Number } \\
\text { of equality } \\
\text { constraints }\end{array}$ & $\begin{array}{l}\text { Number } \\
\text { of inequality } \\
\text { constraints }\end{array}$ \\
\hline MDF & Solver & 201 & 2 & 1 \\
IDF & Optimizer & 203 & 4 & 1 \\
HYBRID & Optimizer & 202 & 3 & 1 \\
NVH & Optimizer & 202 & 2 & 2 \\
\hline
\end{tabular}

\subsection{The larger scale EMA design problem}

A larger scale version of the EMA design problem is proposed in order to extend the benchmarking and investigate the effect of larger scale on the derivative computation techniques and MDO formulations performance. The larger scale EMA design problem consists in implementing an additional discipline that models the trajectory of the actuator. The trajectory is a point-to-point motion profile (Park 1996). Hence, the actuator requirements are a position to reach within a time limit instead of a maximum speed and acceleration. To have a high degree of freedom and differentiable trajectory and speed, it is proposed to use the Fourier series to model the position, the speed, and the acceleration. The mathematical form of these physical variables are sums of sinusoids which guarantee a position, a speed, and an acceleration which are equal to zero at $t=0$ :

$$
\begin{aligned}
A_{\text {ema }}(t)= & \sum_{n=1}^{N} a_{n} \cos (n t)+b_{n} \sin (n t)-a_{n} \\
V_{\text {ema }}(t)= & \sum_{n=1}^{N} a_{n} \frac{1}{n} \sin (n t)-b_{n} \frac{1}{n} \cos (n t) \\
& -a_{n} t+b_{n} \frac{1}{n} \\
X_{\text {ema }}(t)= & \sum_{n=1}^{N}-a_{n} \frac{1}{n^{2}} \cos (n t)-b_{n} \frac{1}{n^{2}} \sin (n t) \\
& -\frac{1}{2} a_{n} t^{2}+b_{n} \frac{1}{n} t .
\end{aligned}
$$

The previous EMA design problem assumed that the actuator had to be designed for short operating time applications for which the thermal performance of the motor is not predominant. Hence, the electromagnetic torque considered was the peak torque and was estimated using a constant external load and an additional inertial load evaluated at the maximum acceleration of the mission profile as depicted in (1). However, for repetitive operations, the temperature increase due to the Joule losses in the windings has to be taken into account. In preliminary design, it is common to choose the root mean square (RMS) torque which indirectly assesses the limitation effect of these losses (Roos et al. 2006). Hence, (1) is replaced by (14).

$T_{\text {em }}=J_{\text {mot }} \cdot A_{\text {rms }} \frac{N_{\text {red }}}{p}+F_{\text {ema }} \frac{p}{N_{\text {red }}}$

where $A_{r m s}$ is the RMS acceleration of the motion profile which can be expressed as follows:

$A_{\text {rms }}=\sqrt{\frac{1}{t_{\text {final }}} \int_{0}^{t_{\text {final }}} A_{\text {ema }}(t)^{2}}$. 
Fig. 10 XDSM diagram for the larger scale EMA design problem

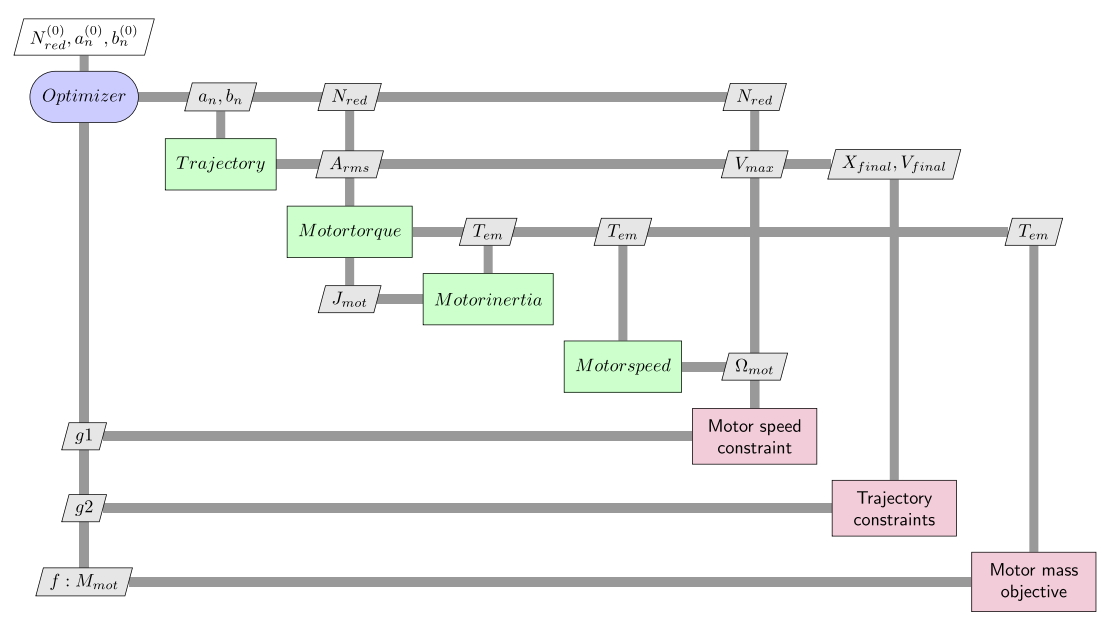

This new evaluation of the electromagnetic torque also requires to choose the continuous torque of the reference component for $T_{e m, r e f}$ instead of its peak torque.

The trajectory model has to be expressed in the form of an optimization problem. The design variables are the Fourier series coefficients $a_{n}$ and $b_{n}$ with a size $N=100$. Two equalities have to be implemented. One ensures that the final position $X_{\text {final }}=0.15 \mathrm{~m}$ is reached at the final time $t_{\text {final }}=1.5 \mathrm{~s}$. The other ensures that the final position is reached at zero speed $V_{\text {final }}=0 \mathrm{~m} \mathrm{~s}^{-1}$ to avoid damaging the mechanical end-stops. To run the trajectory alone, the objective chosen could be to minimize the RMS acceleration of the motion profile.

In the case of the larger scale EMA design problem, this trajectory model is added to the original problem where the objective was to minimize the motor mass. Thus, the optimization problem becomes

$$
\begin{array}{ll}
\text { min. } & M_{\text {mot }} \\
\text { w.r.t } & N_{\text {red }}, a_{n}, b_{n} \\
\text { s.t. } & V_{\text {max }} \cdot \frac{N_{\text {red }}}{p}-\Omega_{\text {mot }} \leq 0 \\
& \left.X_{\text {final }}=0.15\right] \\
& V_{\text {final }}=0 .
\end{array}
$$

The structure of the larger scale EMA design problem is depicted in Fig. 10.

The MDO formulations to solve the multidisciplinary coupling between the motor torque and motor inertia are implemented in exactly the same manner as the ones outlined previously.

An example of resulting trajectory when running an optimization of the larger scale EMA design is shown in Fig. 11.

This resulting trajectory minimizes the motor mass and respects the motor speed limit constraint as well as performing the point-to-point motion profile within the given time.
Table 3 summarizes the characteristics of the larger scale version of the design problem:

All the formulations see themselves added twice 100 design variables as well as 2 equality constraints due to the addition of the trajectory discipline.

\section{Benchmarking of the EMA design problem}

\subsection{Typical MDO formulation benchmarking tests results}

\subsubsection{Tests setup}

The OpenMDAO 2.6.0 version is used for the tests whereas similar tests were performed with OpenMDAO 1.7.3 in Delbecq (2018). The tests are ran for each previous monolithic MDO formulation alongside of two different optimizers of the OpenMDAO optimizers suite. This way
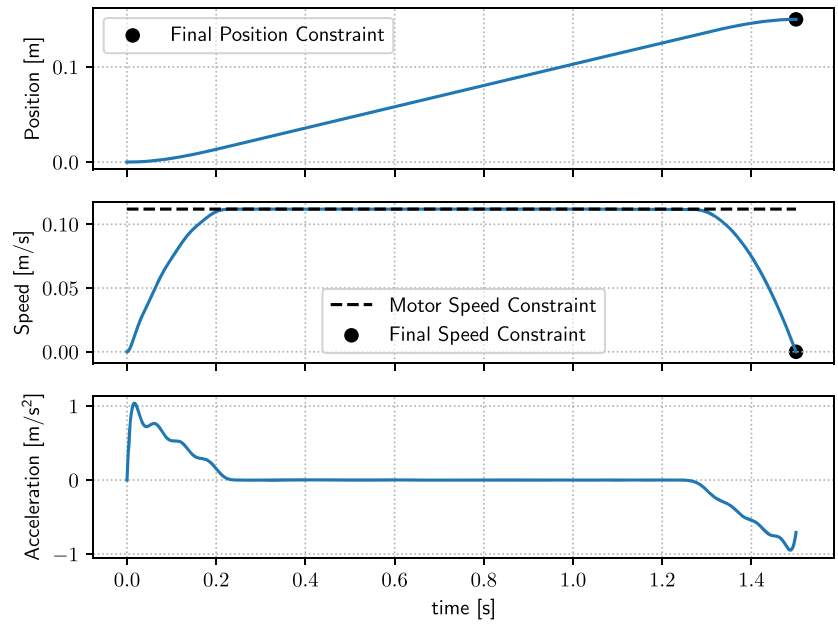

Fig. 11 Example of resulting trajectory when minimizing the motor mass 
Table 3 Formulations characteristics for the EMA design problem

\begin{tabular}{lllll}
\hline Form. & $\begin{array}{l}\text { Solver } \\
\text { type }\end{array}$ & $\begin{array}{l}\text { Number } \\
\text { of design } \\
\text { variables }\end{array}$ & $\begin{array}{l}\text { Number } \\
\text { of equality } \\
\text { constraints }\end{array}$ & $\begin{array}{l}\text { Number } \\
\text { of inequality } \\
\text { constraints }\end{array}$ \\
\hline MDF & Solver & 1 & 0 & 1 \\
IDF & Optimizer & 3 & 2 & 1 \\
HYBRID & Optimizer & 2 & 1 & 1 \\
NVH & Optimizer & 2 & 0 & 2 \\
\hline
\end{tabular}

the Sequential Least Squares Programming (SLSQP) (Kraft 1988) gradient-based optimizer can be compared with the derivative-free COBYLA optimizer (Powell 1994). In addition, different derivative computation methods are used with the SLSQP optimizer since they each conduct to different performances. The methods are referred to as full analytic, semi-analytic, and monolithic derivatives as proposed in the OpenMDAO paper (Gray et al. 2019). The full analytic derivative method uses the analytic derivatives of the model analysis functions (obtained by hand) and the analytic derivatives of the total model (obtained using the MAUD architecture implemented in OpenMDAO (Hwang and Martins 2017; Gray et al. 2019)). The semi-analytic derivative method is similar to the full analytic except that the model analysis functions derivatives are computed numerically (with finite difference (FD) in this study). The monolithic derivative method estimates directly the total system derivatives numerically (with FD in this study) and does not require any knowledge of analysis function derivatives. Therefore, for each MDO formulation, four test configurations are used:

1. SLSQP optimizer with full analytic derivatives

2. SLSQP optimizer with semi-analytic FD derivatives

3. SLSQP optimizer with monolithic FD derivatives

4. COBYLA optimizer (derivative-free)

For the test configurations 3 and 4 , it is considered that no knowledge about the analysis functions is available. Hence, the derivative-free Gauss-Seidel solver is used in the MDF formulation for these configurations.
The tolerance was set to $10^{-6}$. The bounds of the additional design variables that enable coupling consistency are the same for the IDF and HYBRID formulations. The bounds of the global design variables are the same for all formulations. The initial values of variables are the same for each formulation and are set so that the coupling is initially consistent.

As underlined by Gray et al. (2013), there are multiple ways to measure the effectiveness of MDO formulations. In order to standardize the evaluation of these formulations, it is chosen to first use the typical criteria suggested by Gray et al. (2013):

1. Proximity to known solution

2. Total function evaluations

3. Convergence characteristics

\subsubsection{Proximity to known solution}

The values given in Table 4 show the absolute difference between the known optimum and the optimization results for each formulation. MDF, IDF, HYBRID, and NVH converge to the known optimum within the $10^{-6}$ tolerance for the SLSQP with full analytic and semi-analytic FD configurations. However, for the SLSQP with monolithic FD, the HYBRID formulation slightly fails to respect the fixed tolerance. During this last test configuration, MDF and NVH show a small absolute error.

\subsubsection{Total function evaluations}

The number of function and derivative evaluations gives an indication about the computational cost of each formulation. In the MDF formulation, the multidisciplinary coupling is isolated in an OpenMDAO Group with its own system analyzer. Thus, the number of function and derivative evaluations is evaluated on these components for all the formulations. Table 5 summarizes the number of function and derivative evaluations for the different MDO formulations and the test configurations.

It can be depicted that the less costly configuration is the SLSQP optimizer with full analytic derivatives. The lowest
Table 4 Absolute error from known optimum

$M_{m o t}=7.145101$ for all formulations and test configurations solving the EMA design problem

\begin{tabular}{|c|c|c|c|c|}
\hline Formulation & $\begin{array}{l}\text { SLSQP with } \\
\text { full analytic } \\
\text { derivatives }\end{array}$ & $\begin{array}{l}\text { SLSQP with } \\
\text { semi-analytic } \\
\text { FD derivatives }\end{array}$ & $\begin{array}{l}\text { SLSQP with } \\
\text { monolithic } \\
\text { FD derivatives }\end{array}$ & $\begin{array}{l}\text { COBYLA } \\
\text { (derivative-free) }\end{array}$ \\
\hline MDF & 0.000000 & 0.000000 & 0.000000 & 0.000001 \\
\hline IDF & 0.000000 & 0.000000 & 0.000000 & 0.000000 \\
\hline HYBRID & 0.000000 & 0.000000 & 0.000002 & 0.000000 \\
\hline $\mathrm{NVH}$ & 0.000000 & 0.000000 & 0.000000 & 0.000002 \\
\hline
\end{tabular}


Table 5 Number of function evaluations $\#_{f u n}$ and derivative evaluations $\#_{d e r}$ for all formulations and test configurations solving the EMA design problem

\begin{tabular}{|c|c|c|c|c|c|c|c|c|}
\hline \multirow[b]{2}{*}{ Formulation } & \multicolumn{2}{|c|}{$\begin{array}{l}\text { SLSQP with } \\
\text { full analytic } \\
\text { derivatives }\end{array}$} & \multicolumn{2}{|c|}{$\begin{array}{l}\text { SLSQP with } \\
\text { semi-analytic } \\
\text { FD derivatives }\end{array}$} & \multicolumn{2}{|c|}{$\begin{array}{l}\text { SLSQP with } \\
\text { monolithic } \\
\text { FD derivatives }\end{array}$} & \multicolumn{2}{|c|}{$\begin{array}{l}\text { COBYLA } \\
\text { (derivative-free) }\end{array}$} \\
\hline & $\#_{f u n}$ & $\#_{d e r}$ & $\#_{f u n}$ & $\#_{d e r}$ & $\#_{f u n}$ & $\#_{d e r}$ & $\#_{f u n}$ & $\#_{d e r}$ \\
\hline MDF & 24 & 22 & 139 & 22 & 757 & 110 & 186 & 0 \\
\hline IDF & 14 & 14 & 84 & 14 & 168 & 14 & 74 & 0 \\
\hline HYBRID & 9 & 8 & 49 & 8 & 88 & 8 & 587 & 0 \\
\hline $\mathrm{NVH}$ & 8 & 7 & 36 & 7 & 78 & 7 & 26 & 0 \\
\hline
\end{tabular}

computational cost formulation is the $\mathrm{NVH}$, followed by HYBRID, IDF, and MDF. The MDF formulation is greatly penalized by the system analyzer function evaluations. This ranking remains the same for all test configurations. By removing an additional design variable and an additional equality constraint, the HYBRID approach is more effective than the IDF approach. Besides, the fact to transform an equality constraint into an inequality and to reduce the dimension of the additional design variable is very efficient as shown in the NVH results. IDF is also penalized by the dimension of the coupling variables especially the motor inertia which is typically between $1.0 \cdot 10^{-4}$ and $1.0 \cdot 10^{-6}$ $\mathrm{kg} \mathrm{m}^{2}$ for the power required in this application depending if the motor is a high torque or a high speed design. The same effect of motor inertia dimension can be noticed for the HYBRID formulation.

Regarding the gradient-based test configurations, it is clear that the full analytic derivatives is the most efficient followed by the semi-analytic FD and the monolithic FD. This proves the interest of the modular analysis and unified derivatives (MAUD) (Hwang 2015; Hwang and Martins 2017) and the sophistication of the OpenMDAO framework for gradient-based optimization. The full analytic and semianalytic FD test configurations are more efficient than the gradient-free optimizer COBYLA except for the IDF formulation. However, the COBYLA optimizer has a much smaller computational cost than the SLSQP optimizer with monolithic FD for all formulations. Therefore, the choice of derivation computation technique has an evident effect on the number of function evaluations.

\subsubsection{Convergence characteristics}

For ease of reading, the convergence characteristics are given in different figures for each test configuration. The convergence plots represent the value of the relative error between the objective value and the known objective value for each major iteration (optimizer iteration).

Figures 12 and 13 show that the full analytic and semi-analytic FD tests have the same convergence at the optimizer level.
The MDF and NVH formulations converge the most rapidly and are followed by the HYBRID and IDF formulations. However, as shown in Fig. 14, the monolithic FD has a negative effect on the convergence for the MDF whereas it reduces the iteration number for the HYBRID strategy. The IDF and NVH strategies are insensitive to this change of derivative method.

The COBYLA optimizer test configuration results show some slightly different trends. The MDF convergence is the most efficient followed by the NVH and the IDF. This time however the convergence rate of the HYBRID is much slower with over 500 iterations (Fig. 15).

It is important to underline that the choice of the feedforward discipline in the HYBRID formulation influences the effectiveness of the optimization especially when the dimension of the coupling variables are different.

\subsection{Additional tests results}

This section aims to perform some additional tests in order to evaluate the effectiveness of formulations and derivative strategies for common engineering design practices.

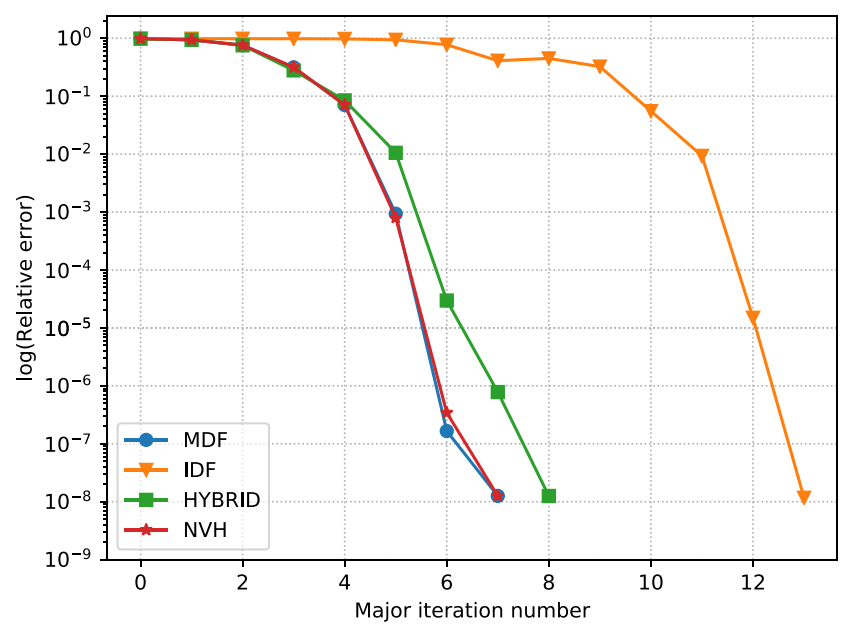

Fig. 12 Relative error vs iteration number for all formulations running the EMA design with the SLSQP optimizer and full analytic derivatives 


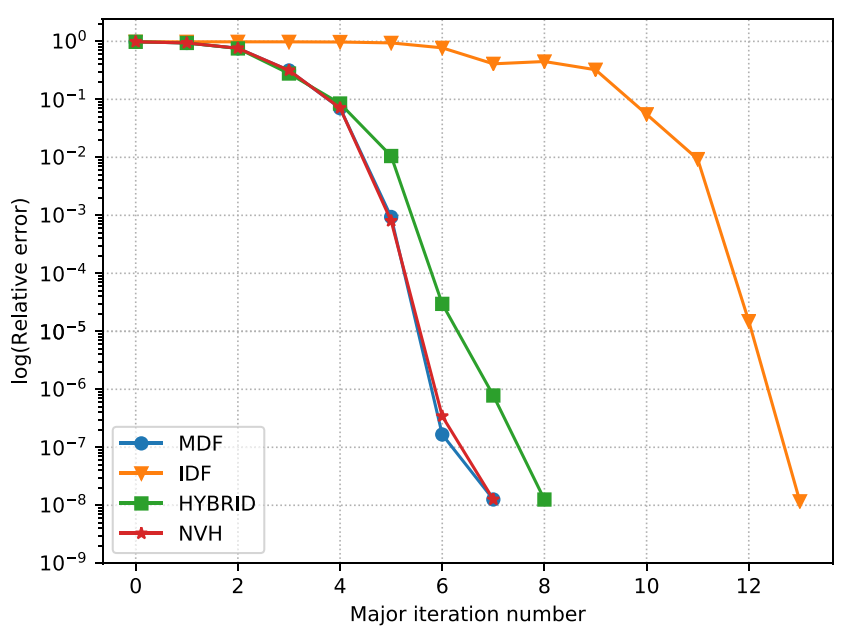

Fig. 13 Relative error vs iteration number for all formulations running the EMA design with the SLSQP optimizer and semi-analytic FD derivatives

\subsubsection{Robustness to initial conditions variations}

In design optimization, it is not always straightforward to establish some initial conditions which will guarantee to find the global optimum in a limited computation time. This is particularly true during preliminary design where the bounds of the design variables have large dimensions. Hence, optimization strategies that offer robustness to the initial conditions can be particularly interesting in engineering design tasks. Therefore, it is proposed to assess such robustness using the following test. The idea is to perform several optimizations with different initial conditions and this for all test cases and formulations proposed in Section 3.1.1. For this purpose, a randomized Latin Hypercube Sampling (LHS) Design of Experiments (DOE) is accomplished for the reducer gear ratio $N_{\text {red }}$, the

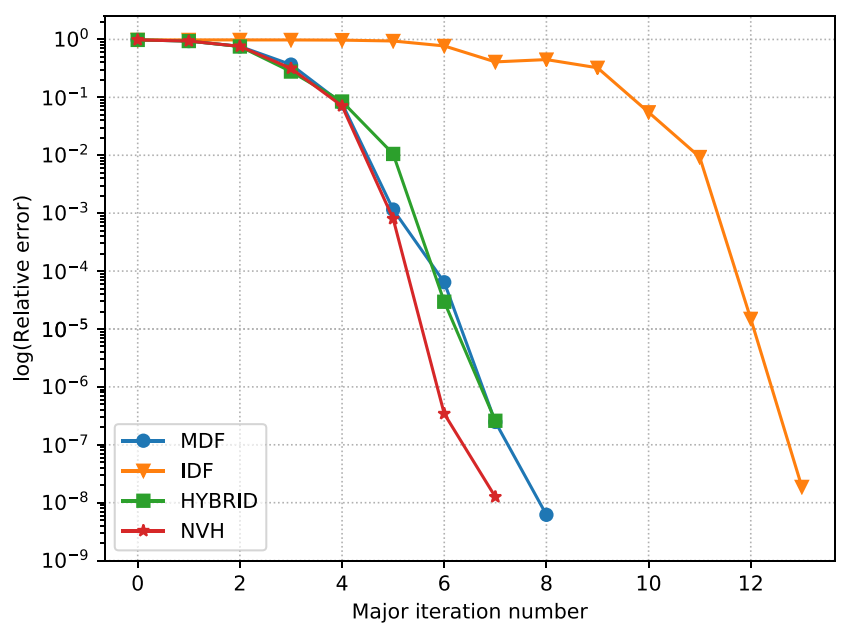

Fig. 14 Relative error vs iteration number for all formulations running the EMA design with the SLSQP optimizer and monolithic FD derivatives

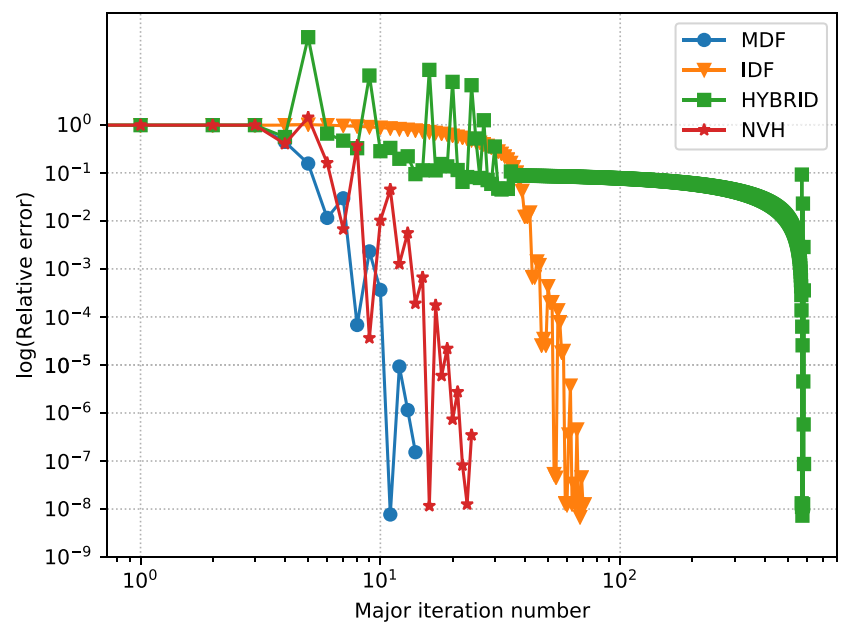

Fig. 15 Relative error vs iteration number for all formulations running the EMA design with the COBYLA optimizer (derivative-free)

design variable, and the motor electromagnetic torque $T_{e m}$ and inertia $J_{m o t}$, the coupling variables. The DOE bounds are the same as in the optimization problem in Section 3.1 and the number of samples is set to 50. To assess the robustness, it is chosen to evaluate the mean, maximum, and minimum number of function evaluations for all formulations and test cases. In addition, the percentage of success in solving the multidisciplinary coupling is given. A test is successful if the constraints are respected and the known objective is found for the given optimizer tolerance $\left(10^{-6}\right)$. The mean, maximum, and minimum values are estimated for only successful samples. The test results of the robustness to initial conditions variations are summarized in Table 6.

The test results show, again, that for this test case, the full analytic derivatives strategy is the most efficient for all MDO formulations to succeed in solving the optimization problem and with the lowest number of function evaluations. The NVH formulation has the smallest mean, maximum, and minimum number of evaluations for all the derivative strategies. The MDF formulation comes second in mean evaluations for the full analytic derivative case but ends just after the HYBRID for the semi-analytic FD case for which the IDF comes last. However, both IDF and HYBRID do not succeed to solve the coupling for all the samples conversely to the MDF. The NVH fails for $12 \%$ of the samples. Hence, the MDF is the most robust formulation regarding initial condition variations. The results show that the monolithic FD derivative strategy has a lower computational cost than the COBYLA optimizer except for the MDF and the IDF. In a general manner, this derivative-free strategy does not show an acceptable robustness to initial conditions variations as most samples end up not successful for all formulations for this test case. 
Table 6 Mean, maximum, and minimum number ([mean - max. - min.]) of function evaluations \# fun $_{\text {and }} \%$ of success for all formulations and test configurations when performing the robustness to initial conditions variations test

\begin{tabular}{|c|c|c|c|c|c|c|c|c|}
\hline \multirow[b]{2}{*}{ Form. } & \multicolumn{2}{|l|}{$\begin{array}{l}\text { SLSQP with } \\
\text { full analytic } \\
\text { derivatives }\end{array}$} & \multicolumn{2}{|l|}{$\begin{array}{l}\text { SLSQP with } \\
\text { semi-analytic } \\
\text { FD derivatives }\end{array}$} & \multicolumn{2}{|l|}{$\begin{array}{l}\text { SLSQP with } \\
\text { monolithic } \\
\text { FD derivatives }\end{array}$} & \multicolumn{2}{|l|}{$\begin{array}{l}\text { COBYLA } \\
\text { (derivative-free) }\end{array}$} \\
\hline & $\#_{f u n}$ & $\begin{array}{l}\% \text { of } \\
\text { success }\end{array}$ & $\#_{f u n}$ & $\begin{array}{l}\% \text { of } \\
\text { success }\end{array}$ & $\#_{f u n}$ & $\begin{array}{l}\% \text { of } \\
\text { success }\end{array}$ & $\#_{f u n}$ & $\begin{array}{l}\% \text { of } \\
\text { success }\end{array}$ \\
\hline MDF & [21.6 - $46-9]$ & 100 & {$[119.7-221-54]$} & 100 & [455.9 - $827-234]$ & 98 & [193.9 - $272-146]$ & 86 \\
\hline IDF & {$[26.5-107-12]$} & 62 & {$[119.8-335-25]$} & 76 & [202.5- $357-48]$ & 66 & {$[212.8-587-52]$} & 34 \\
\hline Hybr. & {$[24.9-107-6]$} & 56 & [89.2 - $380-32]$ & 60 & [152.0 - $632-77]$ & 48 & {$[175.7-587-46]$} & 26 \\
\hline NVH & {$[6.9-9-4]$} & 88 & {$[32.5-41-16]$} & 88 & {$[70.8-89-34]$} & 88 & {$[95.8-865-23]$} & 88 \\
\hline
\end{tabular}

\subsubsection{Robustness to scale variations}

The system design is achieved for a set of requirements. Many products are used for different scales and therefore different values in requirements. For instance in the case of actuation systems, a same family of actuators can contain actuators that move load on a relative scale from $1 \times$ to $30 \times$ depending on the applications. One additional need or request for a design engineer is to have a generic sizing code for all of its product portfolio. This can be made possible for products that have the same physical architecture. In order to evaluate the capability of the different formulations and derivative strategies to fulfill such request, a robustness to scale variations is performed. The test consists in applying a coefficient $k_{F_{e m a}}$ on the actuator external load $F_{\text {ema }}$ to vary the scale of the problem. Thus, (1) becomes

$T_{e m}=J_{m o t} \cdot A_{\max } \frac{N_{\text {red }}}{p}+k_{F_{\text {ema }}} \cdot F_{\text {ema }} \frac{p}{N_{\text {red }}}$.

A set of four samples is chosen which corresponds to different values of $k_{F_{\text {ema }}}: 1,10,20,30$. The optimization problem, the initial values of the variables, and the value of the parameters of the problem are kept identical the typical test results of Section 3.1. The only exception is the upper bounds of the consistency variables $J_{m o t}^{t}$ and $T_{e m}^{t}$ of the IDF and HYBRID formulation that have to be increased so that a technological solution of the electrical motor exists for the increased load value $k_{F_{\text {ema }}} \cdot F_{\text {ema }}$. These new upper bounds are estimated for each sample thanks to a scaling law with respect to $k_{F_{e m a}}$. For each sample of the test, the number of function evaluations is counted and compared for all test cases and formulations proposed in Section 3.1. In this sense, the results are outlined using a bar plot for which the different $k_{F_{e m a}}$ values are indexes as well as the oversizing coefficient $k_{o s}$ with the NVH in order to underline the increasing numerical complexity. The values are set to 0 if the formulation failed solving the multidisciplinary coupling.
Figure 16 shows that the scale variation does not affect significantly the HYBRID and NVH formulations' number of function evaluations when compared with the MDF formulation in the case of full analytic derivatives. Besides, the IDF fails to solve the multidisciplinary coupling for the samples of $k_{F_{e m a}}$ with values of 10,20 , and 30 .

The same observations can be made for Figs. 17 and 18 which correspond to respectively the semi-analytic FD and monolithic FD strategies. Therefore, the derivative computation strategy affects the computational cost linearly with the scale change but does not lead to failures in solving the couplings except the MDF using the monolithic FD.

Figure 19 outlines the test results for the test case of the derivative-free optimizer COBYLA. Here, conclusions are more difficult except that only the $\mathrm{NVH}$ formulation manages to succeed for all the samples. However, it is notable that the number of evaluations increases significantly with the scale change for the NVH which was not the case for the tests with the SLSQP optimizer.

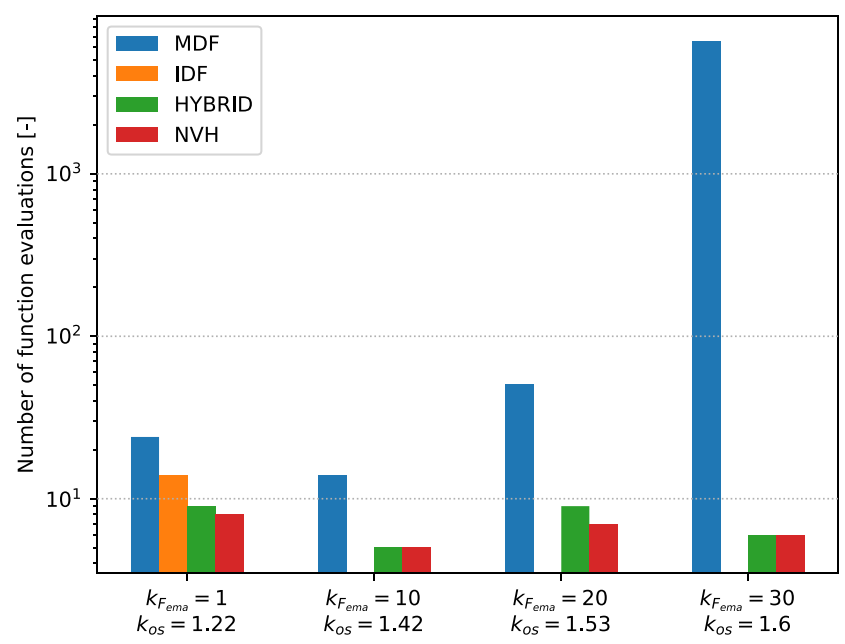

Fig. 16 Number of function evaluations vs scale factor for all formulations running the EMA design with the SLSQP optimizer and full analytic derivatives 


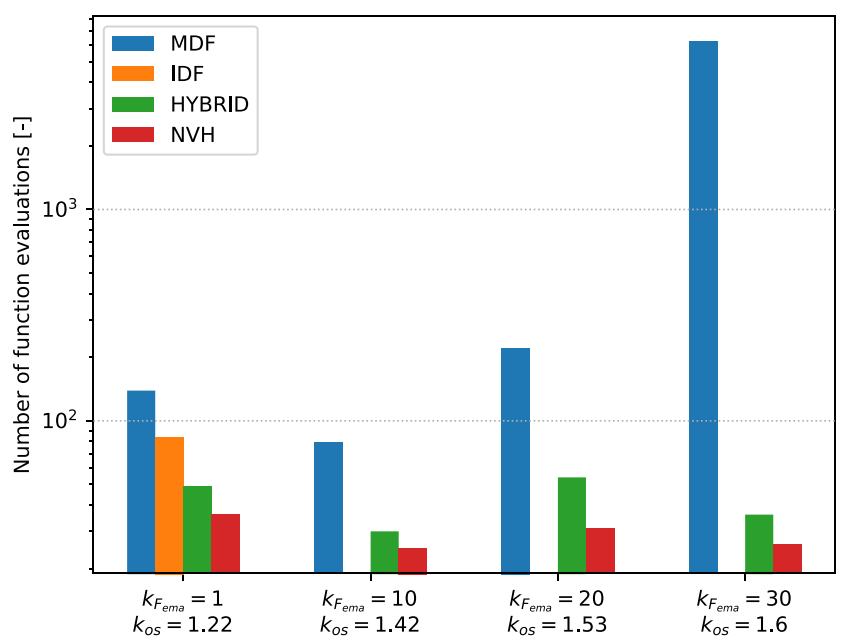

Fig. 17 Number of function evaluations vs scale factor for all formulations running the EMA design with the SLSQP optimizer and semi-analytic FD derivatives

Therefore, for this test case, the HYBRID and NVH formulations are robust to scale variations. Alongside, the MDF formulation remains capable of finding a consistent solution, especially for full analytic and semi-analytic FD, but with an increasing computational cost. The IDF formulation fails when the scale of the load increases which makes it not adapted for the implementation of generic sizing codes. In addition, the utilization of gradient-based optimization is more adequate if a generic sizing code is sought.

\subsubsection{Robustness in design space exploration}

In engineering design, the pure design optimization approach is not adequate for all purposes. One might prefer

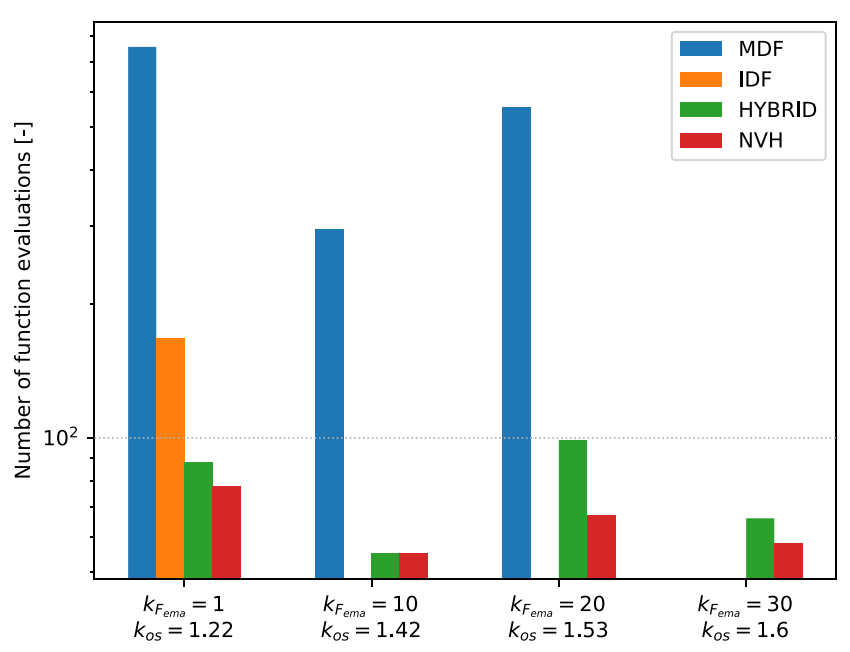

Fig. 18 Number of function evaluations vs scale factor for all formulations running the EMA design with the SLSQP optimizer and monolithic FD derivatives

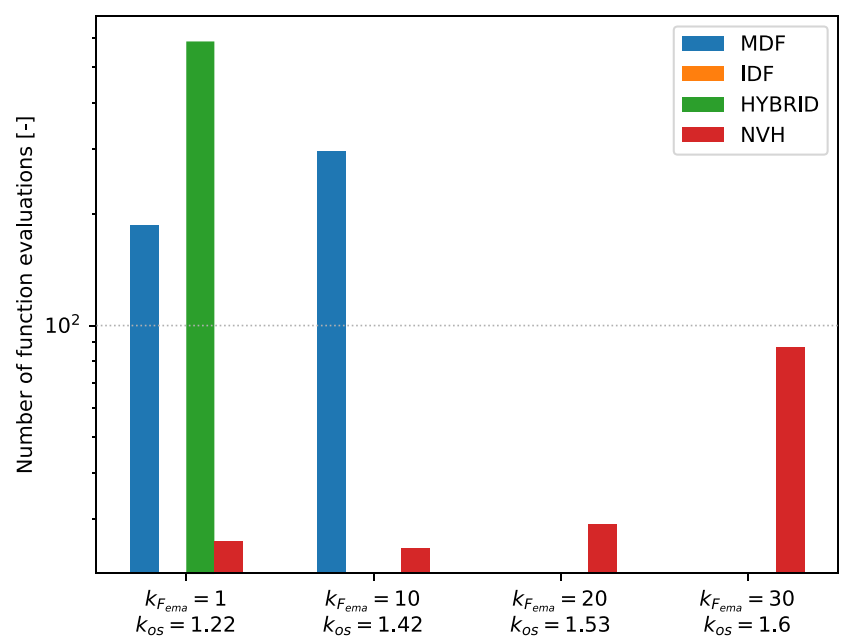

Fig. 19 Number of function evaluations vs scale factor for all formulations running the EMA design with the COBYLA optimizer (derivative-free)

a design space exploration approach in order to better sense the alternatives in the design space rather than obtain one single alternative. Hence, here, a test is ran in order to evaluate the robustness of the different MDO formulations and derivative strategies for the application of design space exploration. This test aims at assessing the capability of the test cases to converge the multidisciplinary coupling, i.e., to perform a Multidisciplinary Design Analysis (MDA), for different configurations in the design space. For this purpose, as achieved for the robustness to initial conditions variations, a randomized LHS DOE is accomplished for the reducer gear ratio $N_{\text {red }}$, the actuator external load $F_{e m a}$, the load maximum acceleration $A_{\max }$, and the ballscrew pitch $p$. For the IDF, HYBRID and NVH formulations consist in running the DOE on top of an optimization problem that performs the MDA. The optimization problem using the $\mathrm{NVH}$ formulation is

$$
\begin{array}{ll}
\text { min. } & M_{\text {mot }} \\
\text { w.r.t } & k_{o s} \\
\text { s.t. } & J_{\text {mot }} \cdot A_{\max } \frac{N_{\text {red }}}{p}+F_{\text {ema }} \frac{p}{N_{\text {red }}}-T_{\text {em }} \leq 0
\end{array}
$$

Conversely, the MDF uses a system analyzer (numerical solver) to perform the MDA. The choice and configuration of the numerical solver depends on the derivative strategy test case as described in Section 3.1. Figure 20 shows the structure of this test for the case of the NVH formulation.

It is important to note that the formulations that use an optimizer to perform the MDA require us to define an objective for the optimizer to solve the consistencies, which is not the primary purpose of design exploration. Defining the wrong objective can lead to inconsistent designs during the exploration. Hence, this can be considered a drawback when compared with the MDF. 
Fig. 20 XDSM diagram for NVH formulation of the EMA design problem

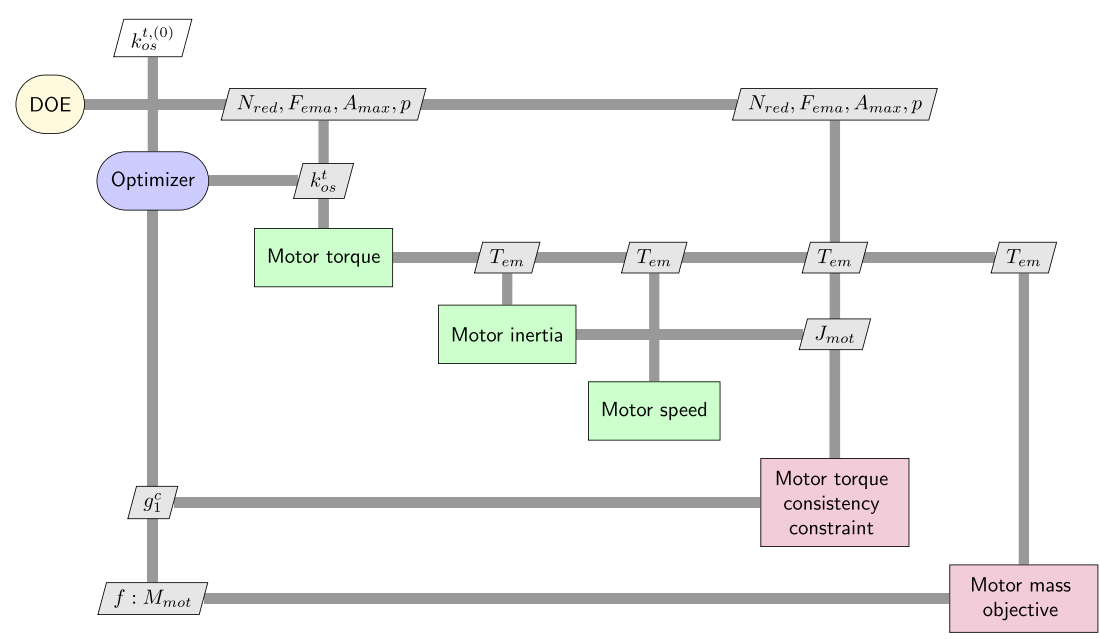

The DOE bounds are the same as in the optimization problem in Section 3.1 for the variable $N_{\text {red }}$ whereas the lower and upper bounds of $F_{e m a}, A_{m a x}$, and $p$ are respectively $\times 0.5$ and $\times 2$ of the fixed value they had in that problem. The number of samples is set to 50. To assess the robustness, it is chosen to evaluate the mean, maximum, and minimum number of function evaluations for all formulations and test cases. In addition, the percentage of success in solving the multidisciplinary coupling is given. The mean value is estimated for only successful samples. A sample is successful if the consistency constraint is respected for the given optimizer tolerance $\left(10^{-6}\right)$. The results of the robustness to design space exploration tests are summarized in Table 7. The MDF formulation is distinguished at the bottom to underline that it does not involve any optimizer.

The results show that at least $6 \%$ of the samples (3 configurations) have no existing solution. For the case that analytic derivatives are used, the most efficient formulation is the NVH. The SLSQP optimizer with full analytic derivatives despite the MDO formulation to perform the
MDA is the most robust compared with the use of the implementation of MDF with the Newton non-linear solver and the direct linear solver. The Newton solver used is the basic one provided by the OpenMDAO framework with no specific line search methods. This corresponds to the comparison of the effectiveness of the optimizer and system analyzer. However, the MDF remains the most efficient in terms of number of functions calls. Conversely to the previous tests, the IDF formulation requires in mean less number of function evaluations than the HYBRID for all test cases except for the COBYLA optimizer case (with a percentage of success of $10 \%$ ). The $\mathrm{NVH}$ formulation shows the best performance for all test cases in terms of robustness and computational cost except the case where analytic derivatives are used as the MDF requests less function calls. Therefore, the most efficient formulation to perform an MDA when analytic derivatives are available is the MDF. Conversely, when derivatives are not available, the NVH formulation is the most efficient for all derivative strategies (semi-analytic FD, monolithic FD, and derivativefree) and is the most robust for all cases.

Table 7 Mean, maximum, and minimum ([mean - max. - min.]) number of function evaluations \# fun $_{\text {and }} \%$ of success for all formulations and test configurations when performing the robustness to design space exploration test on the EMA design problem

\begin{tabular}{|c|c|c|c|c|c|c|c|c|}
\hline \multirow[b]{2}{*}{ Form } & \multicolumn{2}{|l|}{$\begin{array}{l}\text { SLSQP with } \\
\text { full analytic } \\
\text { derivatives }\end{array}$} & \multicolumn{2}{|l|}{$\begin{array}{l}\text { SLSQP with } \\
\text { semi-analytic } \\
\text { FD derivatives }\end{array}$} & \multicolumn{2}{|l|}{$\begin{array}{l}\text { SLSQP with } \\
\text { monolithic FD } \\
\text { derivatives }\end{array}$} & \multicolumn{2}{|l|}{$\begin{array}{l}\text { COBYLA } \\
\text { (derivative-free) }\end{array}$} \\
\hline & $\#_{f u n}$ & $\begin{array}{l}\% \text { of } \\
\text { success }\end{array}$ & $\#_{f u n}$ & $\begin{array}{l}\% \text { of } \\
\text { success }\end{array}$ & $\#_{f u n}$ & $\begin{array}{l}\% \text { of } \\
\text { success }\end{array}$ & $\#_{f u n}$ & $\begin{array}{l}\% \text { of } \\
\text { success }\end{array}$ \\
\hline IDF & {$[4.4-6-4]$} & 74 & {$[26.5-36-24]$} & 78 & {$[52.1-72-48]$} & 66 & [81.4 - $154-31]$ & 28 \\
\hline Hybr. & {$[8.35-39-3]$} & 80 & {$[35.5-73-24]$} & 72 & {$[63.8-157-34]$} & 64 & {$[17.4-19-15]$} & 10 \\
\hline $\mathrm{NVH}$ & {$[3.7-5-2]$} & 92 & {$[16.4-26-11]$} & 94 & {$[35.5-55-23]$} & 94 & {$[14.3-15-13]$} & 70 \\
\hline MDF & [3.1 - 4 - 3] & 44 & {$[18.7-24-18]$} & 52 & {$[18.5-24-18]$} & 46 & {$[15.5-21-11]$} & 28 \\
\hline
\end{tabular}


Table 8 Absolute error from known optimum

$M_{m o t}=15.381369$ for all formulations and test configurations solving the larger scale EMA design problem

\begin{tabular}{|c|c|c|c|c|}
\hline & $\begin{array}{l}\text { SLSQP with } \\
\text { full analytic } \\
\text { derivatives }\end{array}$ & $\begin{array}{l}\text { SLSQP with } \\
\text { semi-analytic } \\
\text { FD derivatives }\end{array}$ & $\begin{array}{l}\text { SLSQP with } \\
\text { monolithic } \\
\text { FD derivatives }\end{array}$ & $\begin{array}{l}\text { COBYLA } \\
\text { (derivative-free) }\end{array}$ \\
\hline Formulation & $\Delta$ Objective & $\Delta$ Objective & $\Delta$ Objective & $\Delta$ Objective \\
\hline $\mathrm{MDF}$ & 0.000000 & 0.000000 & - & - \\
\hline IDF & 0.007275 & 0.007275 & - & - \\
\hline HYBRID & 0.001814 & 0.001814 & - & - \\
\hline $\mathrm{NVH}$ & 0.007111 & 0.007111 & - & - \\
\hline
\end{tabular}

\section{Benchmarking of the larger scale EMA design problem}

Similar to the previous section, this section deals with some typical and additional tests applied to the larger scale EMA design problem which includes the new trajectory discipline. One main remark here is that the tests carried out for the SLSQP optimizer with monolythic FD and the derivative-free COBYLA configurations all fail due to the increased complexity of the problem. This is the case for all four of the proposed MDO formulations. Hence, the results presented will only relate to the full analytic and semianalytic FD derivate computation techniques. In addition, the robustness in design space exploration is not achieved here as speed and acceleration are now determined by the trajectory discipline.

\subsection{Typical MDO formulation benchmarking tests results}

\subsubsection{Proximity to known solution}

The values given in Table 8 show the absolute difference between the known optimum and the optimization results for each formulation. The MDF manages to converge exactly to the global optimum $M_{m o t}=15.381369$. Conversely, the IDF, the HYBRID, and the NVH stop near this optimum leading to non nill, however acceptable, absolute errors.

\subsubsection{Total function evaluations}

As for the original problem, the number of function evaluations is evaluated to assess the computational cost of each configuration. Table 9 summarizes the number of function and derivative evaluations for the different MDO formulations and the test configurations.

The less costly configuration is the SLSQP optimizer with full analytic derivatives. The lowest computational cost formulation is the IDF, followed by the NVH, the HYBRID, and finally the MDF. The MDF formulation is greatly penalized when swapping to semi-analytic FD. Besides, for this derivative computation technique, the $\mathrm{NVH}$ becomes the most performant formulation.

\subsubsection{Convergence characteristics}

The convergence plot given in Fig. 21 is valid for the SLSQP optimizer using full analytic derivatives and semi-analytic FD derivatives test configurations.

All MDO formulations show a similar convergence behavior. It can be noted that the HYBRID and the MDF request more major iterations at the optimizer level. This contributes to the higher computational cost observed previously for both formulations.

These first test results for the larger scale EMA design problem are the opportunity to emphasize the benefit of the MAUD approach. Increasing the scale of the problem leads to failure of the monolythic FD and
Table 9 Number of function evaluations \# fun $_{\text {un }}$ and derivative evaluations $\#_{d e r}$ for all formulations and test configurations solving the larger scale EMA design problem

\begin{tabular}{|c|c|c|c|c|c|c|c|c|}
\hline \multirow[b]{2}{*}{ Formulation } & \multicolumn{2}{|c|}{$\begin{array}{l}\text { SLSQP with } \\
\text { full analytic } \\
\text { derivatives }\end{array}$} & \multicolumn{2}{|c|}{$\begin{array}{l}\text { SLSQP with } \\
\text { semi-analytic } \\
\text { FD derivatives }\end{array}$} & \multicolumn{2}{|c|}{$\begin{array}{l}\text { SLSQP with } \\
\text { monolithic } \\
\text { FD derivatives }\end{array}$} & \multicolumn{2}{|c|}{$\begin{array}{l}\text { COBYLA } \\
\text { (derivative-free) }\end{array}$} \\
\hline & $\#_{f u n}$ & $\#_{d e r}$ & $\#_{f u n}$ & $\#_{d e r}$ & $\#_{f u n}$ & $\#_{d e r}$ & $\#_{f u n}$ & $\#_{d e r}$ \\
\hline MDF & 2858 & 2194 & 13833 & 2194 & - & - & - & - \\
\hline IDF & 780 & 305 & 2305 & 305 & - & - & - & - \\
\hline HYBRID & 1229 & 497 & 3714 & 497 & - & - & - & - \\
\hline $\mathrm{NVH}$ & 895 & 328 & 2207 & 328 & - & - & - & - \\
\hline
\end{tabular}




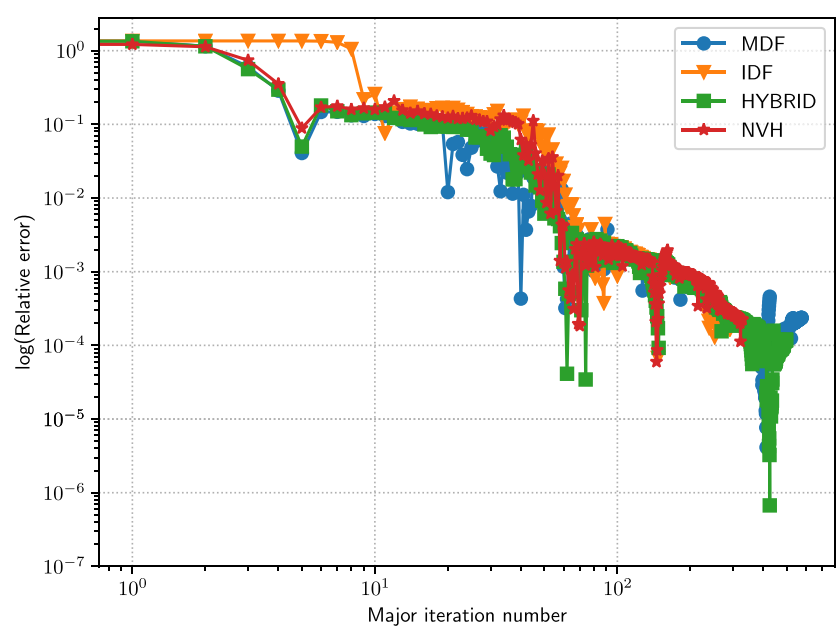

Fig. 21 Relative error vs iteration number for all formulations running the larger scale EMA design problem with the SLSQP optimizer using full analytic derivatives and semi-analytic FD derivatives

derivative-free approaches. Despite that increasing the scale induces a significant increase in the computational cost for the full-analytic and semi-nalytic FD derivative computation techniques, the MAUD approach enables to find a solution. This reminds the adequacy of this approach and the OpenMDAO framework to solve large scale MDAO problems.

\subsection{Additional tests results}

\subsubsection{Robustness to initial conditions variations}

The robustness to initial conditions variations test is performed as for the original EMA design problem in Section 4.2.1 using the same assumptions. The test is driven by variations of the design variables initial conditions except for the ones defining the trajectory, $a_{n}$ and $b_{n}$. Thus, a LHS DOE is accomplished for the reducer gear ratio $N_{\text {red }}$, the design variable, and the motor electromagnetic torque $T_{e m}$ and inertia $J_{m o t}$, the coupling variables. The DOE bounds are the same as in the optimization problem in Section 4.1 and the number of samples is set to 50. The test results of the robustness to initial conditions variations for the larger scale design problem are summarized in Table 10.

The test results show, again, that the full analytic derivatives strategy is the most efficient for all MDO formulations to succeed in solving the optimization problem and with the lowest number of function evaluations. The NVH formulation has the smallest mean, maximum, and minimum number of evaluations for both derivative strategies. The MDF formulation comes last in mean evaluations for the full analytic derivative case but with a better percentage of success. The IDF and HYBRID have a lower percentage of success but also a lower number of function evaluations. The same infers can be outlined for the semi-analytic FD derivatives results.

\subsubsection{Robustness to scale variations}

The robustness to scale variations test is performed as for the original EMA design problem in Section 3.2.2 using the same assumptions. Nevertheless, a smaller range of scale changes is taken since above $10 \times$ the original external load, the complexity of the problem gets too high and results in a failure of all test configurations. Hence, the set of $k_{F_{\text {ema }}}$ coefficients taken to evaluate the robustness to scale change for the larger scale problem is $[1,2,5,10]$.

Figure 22 shows that the scale variation affects the performance of all formulations in terms of number of function evaluations. The MDF fails for a coefficient greater or equal to 2. The failure comes from the MDA numerical solver which is driven by the optimizer into numerically difficult points of the design space. Conversely, the other formulations are successful but show differences between their performances. The effect of scale change on the HYBRID formulation is considerable making it the one that requests the most function evaluations except for $k_{F_{e m a}}=1$

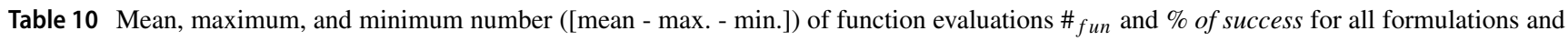
test configurations when performing the robustness to initial conditions variations test for the larger scale design problem

\begin{tabular}{|c|c|c|c|c|c|c|c|c|}
\hline \multirow[b]{2}{*}{ Form } & \multicolumn{2}{|l|}{$\begin{array}{l}\text { SLSQP with } \\
\text { full analytic } \\
\text { derivatives }\end{array}$} & \multicolumn{2}{|l|}{$\begin{array}{l}\text { SLSQP with } \\
\text { semi-analytic } \\
\text { FD derivatives }\end{array}$} & \multicolumn{2}{|l|}{$\begin{array}{l}\text { SLSQP with } \\
\text { monolithic FD } \\
\text { derivatives }\end{array}$} & \multicolumn{2}{|l|}{$\begin{array}{l}\text { COBYLA } \\
\text { (derivative-free) }\end{array}$} \\
\hline & $\#_{f u n}$ & $\begin{array}{l}\% \text { of } \\
\text { success }\end{array}$ & $\#_{f u n}$ & $\begin{array}{l}\% \text { of } \\
\text { success }\end{array}$ & $\#_{f u n}$ & $\begin{array}{l}\% \text { of } \\
\text { success }\end{array}$ & $\#_{f u n}$ & $\begin{array}{l}\% \text { of } \\
\text { success }\end{array}$ \\
\hline MDF & [3145.6 - $4616-1856]$ & 98 & [15028.3 - 22404 - 9247] & 96 & - & 0 & - & 0 \\
\hline IDF & [1129.1 - $2239-623]$ & 86 & [3129.5 - $6388-2093]$ & 76 & - & 0 & - & 0 \\
\hline Hybr. & [1101.7 - 2517 - 618] & 70 & [3351.1 - 6579 - 1597] & 68 & - & 0 & - & 0 \\
\hline $\mathrm{NVH}$ & {$[834.1-1151-623]$} & 92 & [2114.7 - $3123-1491]$ & 92 & - & 0 & - & 0 \\
\hline
\end{tabular}




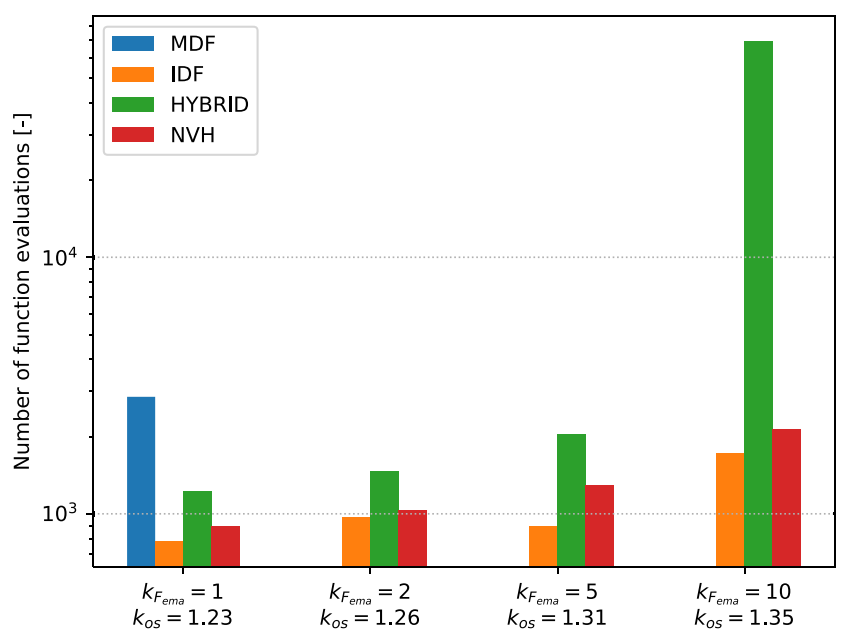

Fig. 22 Number of function evaluations vs scale factor for all formulations running the larger scale EMA design problem with the SLSQP optimizer and full analytic derivatives

where the MDF is more costly. This effect is also notable for the IDF and NVH formulations but to a lesser extent.

For the full analytic derivative computation technique, the IDF is more performant than the NVH. Besides, their number of function evaluations increases with the same proportions with respect to the $k_{F_{e m a}}$ coefficient. However, it can be noted in Fig. 23 that the NVH becomes the most performant when semi-analytic differentiation is used.

Therefore, this test permits to underline that the IDF, HYBRID and NVH formulations are robust to scale variations conversely to MDF. The most performant formulations are the IDF and NVH respectively for the full analytic and the semi-analytic FD derivative computation techniques. When comparing with the results obtained for the original EMA design problem, it can be noted that

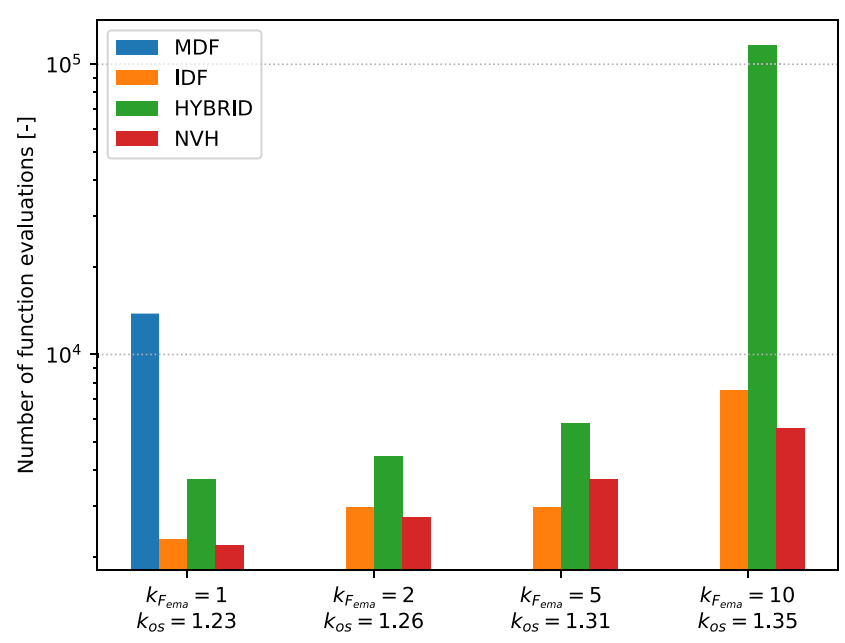

Fig. 23 Number of function evaluations vs scale factor for all formulations running the larger scale EMA design problem with the SLSQP optimizer and semi-analytic FD derivatives there are significant differences when moving to a larger scale optimization problem. The IDF formulation takes the advantage of the degrees of freedom of the trajectory to solve the multidisciplinary coupling between the motor torque and its inertia. This is not the case for the HYBRID and the NVH formulations which see their performances fall with the increase of the number of design variables. Furthermore, this additional degree of freedom is critical for the MDF which has to attempt to solve numerically difficult point of the design space.

\section{Applications}

This section aims at illustrating how the NVH formulation can be extended to other engineering problems. First, the benchmark approach proposed in this paper is applied to the typical MDO test case, the Sellar problem (Sellar et al. 1996), with a particular focus on performance for the optimization and robustness to initial conditions. Then, previous applications of the NVH formulation approach are outlined.

\subsection{Benchmarking the Sellar problem}

The approach taken is extended to the well-known MDO test case: the Sellar problem (Sellar et al. 1996). Many researches in the field of MDO have assessed the performance of monolithic and distributed formulations on this test case (Gray et al. 2013; Ciampa and Nagel 2017). The contribution here is to perform the benchmarking for two additional formulations that is the HYBRID and the NHV. In addition, the derivative strategies comparison includes the monolithic FD. The Sellar problem presents one multidisciplinary coupling that has to be solved by an MDO formulation. Table 11 summarizes the different formulation characteristics for this problem.

\subsubsection{Number of function and derivative evaluations}

The first performance test is a typical optimization with the following initial conditions: $z_{1}=5, z_{2}=2$,

Table 11 Formulations characteristics for the Sellars problem

\begin{tabular}{lllll}
\hline Form & $\begin{array}{l}\text { Solver } \\
\text { type }\end{array}$ & $\begin{array}{l}\text { Number } \\
\text { of design } \\
\text { variables }\end{array}$ & $\begin{array}{l}\text { Number } \\
\text { of equality } \\
\text { constraints }\end{array}$ & $\begin{array}{l}\text { Number } \\
\text { of inequality } \\
\text { constraints }\end{array}$ \\
\hline MDF & Solver & 3 & 0 & 2 \\
IDF & Optimizer & 5 & 2 & 2 \\
HYBRID & Optimizer & 4 & 1 & 2 \\
NVH & Optimizer & 4 & 0 & 3 \\
\hline
\end{tabular}


Table 12 Number of function evaluations $\#_{f u n}$ and derivative evaluations $\#_{d e r}$ for all formulations and test configurations solving the Sellar problem

\begin{tabular}{|c|c|c|c|c|c|c|c|c|}
\hline \multirow[b]{2}{*}{ Formulation } & \multicolumn{2}{|c|}{$\begin{array}{l}\text { SLSQP with } \\
\text { full analytic } \\
\text { derivatives }\end{array}$} & \multicolumn{2}{|c|}{$\begin{array}{l}\text { SLSQP with } \\
\text { semi-analytic } \\
\text { FD derivatives }\end{array}$} & \multicolumn{2}{|c|}{$\begin{array}{l}\text { SLSQP with } \\
\text { monolithic } \\
\text { FD derivatives }\end{array}$} & \multicolumn{2}{|c|}{$\begin{array}{l}\text { COBYLA } \\
\text { (derivative-free) }\end{array}$} \\
\hline & $\#_{f u n}$ & $\#_{d e r}$ & $\#_{f u n}$ & $\#_{d e r}$ & $\#_{f u n}$ & $\#_{d e r}$ & $\#_{f u n}$ & $\#_{d e r}$ \\
\hline $\mathrm{MDF}$ & 21 & 20 & 105 & 20 & 184 & 31 & 233 & 0 \\
\hline IDF & 27 & 18 & 99 & 18 & (93) & (14) & (179) & $(0)$ \\
\hline HYBRID & 6 & 6 & 30 & 6 & 30 & 5 & 65 & 0 \\
\hline $\mathrm{NVH}$ & 7 & 6 & 31 & 6 & 31 & 5 & 83 & 0 \\
\hline
\end{tabular}

$y=0, x=5$. The tests were ran for the same four test configurations as the EMA design problem which are presented in Section 3.1.1. The results for each test case in terms of number of function and derivative evaluations are outlined in Table 12.

The results show that the ranking in terms of computational cost are similar to the EMA design problem. The HYBRID is slightly in front of the NVH, followed by the IDF and the MDF. An exception can be noted for the full analytic derivatives strategy where the MDF required less function calls than the IDF. It is important to note that the IDF fails to find the exact optimum for the monolithic FD and the COBYLA optimizer despite that all the constraints are respected. Furthermore, it is possible to observe that for the HYBRID and the NVH formulations, the computational cost of the semi-analytic and monolithic FD is the same whereas for the other formulations and the EMA design problem, the monolithic strategy was the most costly. Finally, a last observation can be made regarding the comparison between monolithic FD and the COBYLA optimizer. The monolithic is less costly than the optimization with COBYLA for the Sellar problem conversely to the EMA design problem.

\subsubsection{Robustness to initial conditions variations}

Similarly to the test achieved for the EMA design problem in Section 4.2.1, several optimizations with different initial conditions are performed for the four test configurations and all monolithic formulations. For this purpose, a randomized LHS DOE is accomplished for $x \in[0-10], z_{1} \in[-10-$ $10]$, and $z_{2} \in[0-10]$. The results for each test case in terms of mean, maximum, and minimum number of function evaluations as well as the percentage of success are shown in Table 13.

In a general manner, this test shows that the Sellar problem is difficult to solve for a large part of the design space. The MDF formulation is the less efficient during this test on initial condition variations. It presents the least percentage of success and the highest mean number of function evaluations for all the test configurations except for the monolithic FD and COBYLA cases where the percentage of success is lower for IDF and HYBRID. The HYBRID and the NVH are the formulations that request the less function evaluations. However, their percentage of success is lower than the IDF for the full analytic and semianalytic FD derivative strategies. The percentages of success

Table 13 Mean, maximum, and minimum number ([mean - max. - min.]) of function evaluations $\#_{f u n}$ and $\%$ of success for all formulations and test configurations when performing the robustness to initial conditions variations test to the Sellar problem

\begin{tabular}{|c|c|c|c|c|c|c|c|c|}
\hline \multirow[b]{2}{*}{ Form. } & \multicolumn{2}{|l|}{$\begin{array}{l}\text { SLSQP with } \\
\text { full analytic } \\
\text { derivatives }\end{array}$} & \multicolumn{2}{|l|}{$\begin{array}{l}\text { SLSQP with } \\
\text { semi-analytic } \\
\text { FD derivatives }\end{array}$} & \multicolumn{2}{|l|}{$\begin{array}{l}\text { SLSQP with } \\
\text { monolithic FD } \\
\text { derivatives }\end{array}$} & \multicolumn{2}{|l|}{$\begin{array}{l}\text { COBYLA } \\
\text { (derivative-free) }\end{array}$} \\
\hline & $\#_{f u n}$ & $\begin{array}{l}\% \text { of } \\
\text { success }\end{array}$ & $\#_{f u n}$ & $\begin{array}{l}\% \text { of } \\
\text { success }\end{array}$ & $\#_{f u n}$ & $\begin{array}{l}\% \text { of } \\
\text { success }\end{array}$ & $\#_{f u n}$ & $\begin{array}{l}\% \text { of } \\
\text { success }\end{array}$ \\
\hline MDF & [28.6 - $48-20]$ & 40 & [145.5 - $316-105]$ & 34 & [294.3 - 948 - 208] & 36 & {$[240.7-332-184]$} & 42 \\
\hline IDF & {$[27.4-90-6]$} & 58 & [85.6 - $140-35]$ & 54 & [118.1 - $301-43]$ & 22 & [91.9 - $115-73]$ & 30 \\
\hline Hybr. & {$[6.9-9-5]$} & 48 & {$[33.7-45-25]$} & 46 & {$[36.5-45-25]$} & 46 & [81.25 - $114-70]$ & 32 \\
\hline $\mathrm{NVH}$ & {$[15.7-142-6]$} & 54 & [57.7 - $93-30]$ & 50 & {$[58.3-93-30]$} & 44 & [88.2 - $179-56]$ & 56 \\
\hline
\end{tabular}




\begin{tabular}{|c|c|c|c|c|}
\hline \multirow{2}{*}{ Application } & \multicolumn{3}{|c|}{ Number of } & \multirow{2}{*}{ Disciplines } \\
\hline & Variables & Constraints & Couplings & \\
\hline Spoiler linear EMA & 11 & 4 & 3 & $\begin{array}{l}\text { Response surface of transient lumped } \\
\text { parameter simulation / Kinematics / } \\
\text { Electromechanics / Heat Transfer }\end{array}$ \\
\hline Aileron rotary EMA & 12 & 10 & 2 & $\begin{array}{l}\text { Surrogate model of FEM for Heat } \\
\text { Transfer / Surrogate model of FEM for } \\
\text { CFD / Surrogate model of FEM for } \\
\text { Electromagnetics / Surrogate model of } \\
\text { FEM for Structural Mechanics }\end{array}$ \\
\hline $\mathrm{DC} / \mathrm{DCC}$ & 7 & 6 & 2 & $\begin{array}{l}\text { Surrogate model of FEM for Heat } \\
\text { Transfer / Surrogate model of FEM for } \\
\text { Electromagnetics / Power Electronics }\end{array}$ \\
\hline Primary Flight Control Actuation System & 7 & 12 & 1 & $\begin{array}{l}\text { Surrogate model of FEM for: Heat } \\
\text { Transfer, CFD, Electromagnetics, } \\
\text { Structural Mechanics / Power } \\
\text { Electronics / Mechanical Design }\end{array}$ \\
\hline Electric Thrust Reverser Actuation System & 8 & 9 & 2 & $\begin{array}{l}\text { Response surface of transient lumped } \\
\text { parameter simulation / Power } \\
\text { Electronics / Electromechanics / } \\
\text { Trajectory Optimization }\end{array}$ \\
\hline
\end{tabular}

Fig. 24 Summary of the applications using the NVH formulation

of each formulation remain relatively close to each other. Hence, this test case is not the best to prove the robustness of each formulation when compared with the EMA design case. Nevertheless, the computational efficiency observations lead to the same ranking of formulations as the previous test.

\subsection{Recent applications of the NVH formulation}

The NVH formulation has been employed in more complex problems especially for actuation system design applications. The design optimization of an EMA for spoiler flight control surfaces was achieved using the $\mathrm{NVH}$ formulation with the particularity of using a response surface of dynamic lumped parameter simulations to estimate the effects of 2D kinematics on the actuator loads (Budinger et al. 2014). A similar approach was then used in addition to surrogate models of FEM simulations used to assess the thermal behavior in a confined space of an aileron rotary EMA (Sanchez and Delbecq 2016). The same methodology was applied to the design of a DC/DC power converter where there where multidisciplinary couplings between the component choice and the assessment of their temperature (Sanchez et al. 2017a). Similar work was achieved for a linear EMA architecture with the specificity of using a co-simulation Functional Mock-up Unit for the thermal response of the actuator (Delbecq et al. 2017). More complex systems were then addressed with a higher number of analysis functions (over 100 equations) such as a primary flight control actuation system (Delbecq et al. 2018b; Delbecq 2018) and an electrical thrust reverser actuation system (Delbecq et al. 2018a; Delbecq 2018). A summary of the characteristics of these applications is given in Fig. 24.

Therefore, despite that the NVH requires to modify some models, an additional variable and inequality constraint, it can be adapted to most engineering problems. The modification effort has to be compared with the significant robustness and computational efficiency this formulation may lead to when compared with more classic monolithic formulations.

\section{Conclusions}

The main purpose of this paper is the simultaneous benchmark of monolithic formulations and derivative computation techniques using the OpenMDAO framework. 
For this purpose, a new test problem was used. This test problem corresponds to the sizing problem of an high dynamic EMA for thrust vector control applications. The particularity of this problem is that it is possible to increase the strength of the coupling by scaling the actuator external load. This leads to numerical difficulties as the solution can be non unique or absent making the problem original.

The problem implementation was then presented for each of the studied formulations: MDF, IDF, HYBRID, and NVH. The new NVH formulation was presented; it consisted of modifying an equation by adding an over-sizing coefficient and setting the former equation as an inequality constraint leading to two design variables and two inequality constraints.

The benchmarking included the comparison of four different derivative computation techniques. The full analytic strategy used analytic equations to compute the partial derivatives of the analysis functions and the MAUD approach to compute the total derivatives. Similarly, the semi-analytic strategy used FD to compute the partial derivatives of the analysis functions. The monolithic FD used FD to compute directly the total derivatives of the system. Finally, the derivative free strategy was illustrated by using the COBYLA optimizer.

To achieve the benchmarking, typical performance criteria were used such as the absolute error with known optimum, the number of function evaluations, and the convergence characteristics. This paper also proposed some original performance criteria that focused on robustness. The first criteria was the capability of the configurations to find the global optimum when varying the initial conditions. Another test was achieved to assess the robustness to solve the multidisciplinary coupling when scaling up the problem and thus the numerical complexity. The last test enabled to assess the design exploration capacities of the configurations to solve the multidisciplinary couplings when running an MDA in a larger design space.

The benchmarking results have shown that the most efficient configuration was dependent on the criteria. Regarding computational cost, the HYBRID and the $\mathrm{NVH}$ formulations outperform the IDF and the MDF. Regarding robustness, the NVH is the best choice except for the variation of initial conditions where the MDF succeeded totally. Therefore, in a general manner, the most computationally efficient and robust configuration is the $\mathrm{NVH}$ formulation.

The benchmarking of the larger scale EMA design problem enables to infer on additional aspects. The main result is that the monolythic FD and derivative-free techniques fail in solving the problem due to the increase of the number of design variables and the complexity introduce by the trajectory analysis. Hence, this recalls the benefit of the MAUD approach for large-scale MDAO. An other conclusion is that the performance of the MDO formulations changes when compared with the original problem. Here, the IDF and the NVH are the two formulations that show the best computational cost and robustness.

Using the same benchmark approach to the Sellar problem enabled to outline that the problem is strongly dependent on the initial conditions. Furthermore, it showed the interest of a using novel test problem as the EMA design problem since the Sellar did not lead to significant difference between the derivative computation techniques in terms of robustness performance.

This benchmark has also shown that the HYBRID formulation is worth looking into for small-scale problem as it is in most cases simpler and more efficient than the IDF and does not require any model manipulation conversely to the NVH. Indeed, the NVH formulation's main disadvantage is that this approach requires a modification of the governing equations which makes it less generic and more invasive than traditional MDO formulations. Finally, the most efficient and robust for all the formulations was the full analytic strategy which is encouraging for research on efficient gradient-based optimization especially for large scale problem.

Acknowledgments The authors would like to acknowledge Safran and the French National Association of Research and Technology who funded the PhD thesis that produced most of the work presented in this paper.

\section{Compliance with ethical standards}

Conflict of interest The authors declare that they have no conflict of interest.

Replication of results The results presented in this paper were generated by the source code available at the following Github repository: https://github.com/SizingLab/RoR_SAMO_paper_Benchmarking_ monolythic_MDO_formulations.

\section{References}

Alexandrov N, Lewis R (2000) Algorithmic perspectives on problem formulations in mdo. In: 8th symposium on multidisciplinary analysis and optimization, pp 4719

Balling RJ, Sobieszczanski Sobieski J (1996) Optimization of coupled systems-a critical overview of approaches. AIAA J 34(1):6-17

Beiranvand V, Hare W, Lucet Y (2017) Best practices for comparing optimization algorithms. Optim Eng 18(4):815-848

Budinger M, Reysset A, Halabi TE, Vasiliu C, Mare JC (2014) Optimal preliminary design of electromechanical actuators. Proc Inst Mech Eng Part G J Aer Eng 228(9):1598-1616

Bunch JR, Hopcroft JE (1974) Triangular factorization and inversion by fast matrix multiplication. Math Comput 28(125):231-236

Carnevale C, Resta P (2007) Vega electromechanical thrust vector control development. In: 43rd AIAA/ASME/SAE/ASEE joint propulsion conference \& exhibit, pp 5812 
Chauhan SS, Hwang JT, Martins JR (2017) Benchmarking approaches for the multidisciplinary analysis of complex systems using a taylor series-based scalable problem. In: World congress of structural and multidisciplinary optimisation, Springer, pp 98-116

Chell BW, Hoffenson S, Blackburn MR (2019) A comparison of multidisciplinary design optimization architectures with an aircraft case study. In: AIAA Scitech 2019 Forum, pp 0700

Ciampa PD, Nagel B (2017) The agile paradigm: the next generation of collaborative mdo. In: 18th AIAA/ISSMO multidisciplinary analysis and optimization conference, pp 4137

Delbecq S (2018) Knowledge-based multidisciplinary sizing and optimization of embedded mechatronic systems - application to aerospace electro-mechanical actuation systems. $\mathrm{PhD}$ thesis, Toulouse, INSA

Delbecq S, Budinger M, Hazyuk I, Sanchez F, Piaton J (2017) A framework for sizing embedded mechatronic systems during preliminary design. IFAC-PapersOnLine 50(1):4354-4359

Delbecq S, Budinger M, Piaton J (2018a) A more integrated design approach for embedded mechatronic systems: application to electrical thrust reverser actuation systems. In: ICAS 2018 congress of the international council of the aeronautical sciences

Delbecq S, Budinger M, Piaton J, Dagusé B (2018b) Optimization of primary flight control actuation system using parametric sizing models of actuators, power electronics and structural analysis. Recent Advances in Aerospace Actuation Systems and Components, INSA Toulouse, pp 132-138

Gray J, Moore KT, Hearn TA, Naylor BA (2013) Standard platform for benchmarking multidisciplinary design analysis and optimization architectures. AIAA J 51(10):2380-2394

Gray JS, Hwang JT, Martins JR, Moore KT, Naylor BA (2019) Openmdao: an open-source framework for multidisciplinary design, analysis, and optimization. Struct Multidisc Optim 59:1075-1104

Hwang JT (2015) A modular approach to large-scale design optimization of aerospace systems. University of Michigan, $\mathrm{PhD}$ thesis

Hwang JT, Martins J (2017) A computational architecture for coupling heterogeneous numerical models and computing coupled derivatives. ACM Transactions on Mathematical Software

Kessler C (2011) Active rotor control for helicopters: individual blade control and swashplateless rotor designs. CEAS Aeronaut $\mathrm{J}$ 1(1-4):23

Kodiyalam S, Sobieszczanski-Sobieski J (2001) Multidisciplinary design optimization-some formal methods, framework requirements, and application to vehicle design. Int J Veh Des 25(1-2):322

Kraft D (1988) A software package for sequential quadratic programming. Forschungsbericht- Deutsche Forschungs- und Versuchsanstalt fur Luft- und Raumfahrt

Lambe AB, Martins JRRA (2012) Extensions to the design structure matrix for the description of multidisciplinary design, analysis, and optimization processes. Struct Multidiscip Optim 46:273-284. https://doi.org/10.1007/s00158-012-0763-y
Martins JR, Hwang JT (2013) Review and unification of methods for computing derivatives of multidisciplinary computational models. AIAA J 51(11):2582-2599

Martins JR, Lambe AB (2013) Multidisciplinary design optimization: a survey of architectures. AIAA J 51(9):2049-2075

Maskrey R, Thayer W (1978) A brief history of electrohydraulic servomechanisms. Moog Technical Bulletin, pp 141

Papalambros PY, Wilde DJ (2000) Principles of optimal design: modeling and computation. Cambridge University Press, Cambridge

Park J (1996) Motion profile planning of repetitive point-topoint control for maximum energy conversion efficiency under acceleration conditions. Mechatronics 6(6):649-663

Perez R, Liu H, Behdinan K (2004) Evaluation of multidisciplinary optimization approaches for aircraft conceptual design. In: 10th AIAA/ISSMO multidisciplinary analysis and optimization conference, pp 4537

Powell MJ (1994) A direct search optimization method that models the objective and constraint functions by linear interpolation. In: Advances in optimization and numerical analysis, Springer, pp 51-67

Reysset A, Budinger M, Maré JC (2015) Computer-aided definition of sizing procedures and optimization problems of mechatronic systems. Concurr Eng 23(4):320-332

Roos F, Johansson H, Wikander J (2006) Optimal selection of motor and gearhead in mechatronic applications. Mechatronics 16(1):63-72

Sanchez F, Delbecq S (2016) Surrogate modeling technique for the conceptual and preliminary design of embedded actuation systems and components. In: International congress of the aeronautical sciences

Sanchez F, Budinger M, Delbecq S, Hazyuk I (2017a) Modelling and design approaches for the preliminary design of power electronic converters. In: ELECTRIMACS

Sanchez F, Budinger M, Hazyuk I (2017b) Dimensional analysis and surrogate models for the thermal modeling of multiphysics systems. Appl Therm Eng 110:758-771

Sellar R, Batill S, Renaud J (1996) Response surface based, concurrent subspace optimization for multidisciplinary system design. In: 34th aerospace sciences meeting and exhibit, pp 714

Stefano Bianchi E (2005) The launchers and the vega programme and the launcher subsystems http://www.ingaero.uniroma1.it/ attachments/1609_VEGA\%20\%20sapienza\%20small.pdf

Tedford NP, Martins JR (2010) Benchmarking multidisciplinary design optimization algorithms. Optim Eng 11(1):159183

Thareja R, Haftka R (1986) Numerical difficulties associated with using equality constraints toachieve multi-level decomposition in structural optimization. In: 27th structures, structural dynamics and materials conference, pp 854

Publisher's note Springer Nature remains neutral with regard to jurisdictional claims in published maps and institutional affiliations. 